ESAIM: M2AN 51 (2017) 115-145

DOI: $10.1051 / \mathrm{m} 2 \mathrm{an} / 2016018$
ESAIM: Mathematical Modelling and Numerical Analysis

www.esaim-m2an.org

\title{
FORMAL DEDUCTION OF THE SAINT-VENANT-EXNER MODEL INCLUDING ARBITRARILY SLOPING SEDIMENT BEDS AND ASSOCIATED ENERGY
}

\author{
Enrique D. Fernández-Nieto ${ }^{1}$, Tomás Morales de Luna ${ }^{2}$, Gladys \\ Narbona-Reina ${ }^{1}$ and JeAn de Dieu Zabsonré ${ }^{3}$
}

\begin{abstract}
In this work we present a deduction of the Saint-Venant-Exner model through an asymptotic analysis of the Navier-Stokes equations. A multi-scale analysis is performed in order to take into account that the velocity of the sediment layer is smaller than the one of the fluid layer. This leads us to consider a shallow water type system for the fluid layer and a lubrication Reynolds equation for the sediment one. This deduction provides some improvements with respect to the classic Saint-VenantExner model: (i) the deduced model has an associated energy. Moreover, it allows us to explain why classic models do not have an associated energy and how they can be modified in order to recover a model with this property. (ii) The model incorporates naturally a necessary modification that must be taken into account in order to be applied to arbitrarily sloping beds. Furthermore, we show that in general this modification is different from the ones considered classically. Nevertheless, it coincides with a classic one in the case of constant free surface. (iii) The deduced solid transport discharge naturally depends on the thickness of the moving sediment layer, which allows to ensure sediment mass conservation. Moreover, we include a simplified version of the model for the case of quasi-stationary regimes. Some of these simplified models correspond to a generalization of classic ones such as Meyer-Peter and Müller and Ashida-Michiue models. Three numerical tests are presented to study the evolution of a dune for several definition of the repose angle, to see the influence of the proposed definition of the effective shear stress in comparison with the classic one, and by comparing with experimental data.
\end{abstract}

Mathematics Subject Classification. 35Q30, 35Q35, 35Q70, 35Q86, 65Z02, 76D05, 76D09, 86А05, 93A 30 .

Received July 14, 2015. Revised December 23, 2015. Accepted March 8, 2016.

\section{INTRODUCTION}

The Saint-Venant-Exner system (see [17]) is generally used to model the bedload transport in rivers, lakes and coastal areas. Sediment transport is usually divided into three types: surface creep, saltation and suspension.

Keywords and phrases. Saint-Venant-Exner, bedload, Reynolds equation.

1 Dpto. Matemática Aplicada I, ETS Arquitectura, Universidad de Sevilla, Avda. Reina Mercedes N. 2, 41012 Sevilla, Spain. edofer@us.es; gnarbona@us.es

2 Dpto. de Matemáticas, Universidad de Córdoba, Campus de Rabanales, 14071 Córdoba, Spain. tomas.morales@uco.es

3 Institut Universitaire de Technologie, Université Polytechnique de Bobo-Dioulasso, 01, BP 1091 Bobo-Dioulasso 01, Burkina

Faso. jzabsonre@gmail.com 
Surface creep is defined as the type of transport where sediment grains roll or slide along the bed. Saltation transport is defined as the type of transport where single grains jump over the bed a length proportional to their diameter, losing for instants the contact with the soil. Sediment is suspended when the flux is intense enough so that the sediment grains reach height over the bed. There is not a clear distinction between surface creep and saltation, so that these types of transport are usually called bedload transport. At low Froude numbers, the bedload is the dominating transport mechanism which is the regime under study in this paper.

The Saint-Venant-Exner system (SVE in what follows) is defined in terms of a hydrodynamical component coupled with a morphodynamical one. The hydrodynamical component in most cases is modeled by the SaintVenant system. Although it is not necessarily a good model for the hydrodynamic component, it is widely used for its simplicity and because it is valid in a wide range of applications. The equation that describe the morphodynamical component is the well known Exner equation, that is a continuity equation. Then, the Saint-Venant-Exner model can be written under the following form:

$$
\left\{\begin{array}{l}
\partial_{t} h_{1}+\operatorname{div}_{x} q_{1}=0, \\
\partial_{t} q_{1}+\operatorname{div}_{x}\left(\frac{q_{1} \otimes q_{1}}{h_{1}}\right)+\nabla_{x}\left(\frac{1}{2} g h_{1}^{2}\right)+g h_{1} \nabla_{x}\left(h_{2}+b\right)+\tau / \rho_{1}=0, \\
\partial_{t} h_{2}+\operatorname{div}_{x} q_{b}=0,
\end{array}\right.
$$

where $x=\left(x_{1}, x_{2}\right)$ is the horizontal spacial coordinates, $t$ represents the time variable, $q_{1}=h_{1}(x, t) u_{1}(x, t)$ represents the water discharge, $h_{1}(x, t)$ the thickness of the fluid and $u_{1}=\left(u_{1[1]}, u_{1[2]}\right)$ its horizontal velocity. $\tau$ is the shear stress at the bottom and $\rho_{1}$ the density of the fluid. The unknown function $h_{2}=h_{2}(x, t)$ is the thickness of the sediment layer (see Fig. 1) and $q_{b}$ denotes the solid transport discharge. $g$ is the gravity constant and $b$ is the fixed bottom, usually called the bedrock layer. In what follows we shall denote $\eta=b+h_{2}$, being $z=\eta(x, t)$ the sediment bed surface.

To close the system, it is necessary to define the solid transport discharge $q_{b}$ as well as the friction term $\tau$. Several formulae for $q_{b}$ can be found in the literature. For example the classic formula proposed by Grass [29] assumes that the movement of the sediment begins at the same time as for the fluid and both move in the same direction. It is defined by $q_{b}=A_{g}\left|u_{1}\right|^{m_{g}-1} u_{1}$ where $A_{g}$ is a constant which takes into account the grain size and the kinematic viscosity and $m_{g}$ is a positive real number, such that $1 \leq m_{g} \leq 4$. Nevertheless, for practical applications, some other types of formulae have been proposed in the literature, for instance, by Meyer-Peter and Müller [38], Van Rijn's [52], Einstein [16], Nielsen [42], Fernández-Luque and Van Beek [6,20], Ashida and Michiue [1], Engelund and Fredsoe [18], Kalinske [32] or Charru [9]. Such formulae are usually presented in nondimensional form and can be written as follows,

$$
\frac{q_{b}}{Q}=\operatorname{sgn}(\tau) \frac{k_{1}}{(1-\varphi)} \theta^{m_{1}}\left(\theta-k_{2} \theta_{c}\right)_{+}^{m_{2}}\left(\sqrt{\theta}-k_{3} \sqrt{\theta_{c}}\right)_{+}^{m_{3}},
$$

where $Q$ represents the characteristic discharge, $Q=d_{s} \sqrt{g(1 / r-1) d_{s}}, r$ is the density ratio, $r=\rho_{1} / \rho_{2}$, being $\rho_{2}$ the density of the sediment particles and $d_{s}$ the mean diameter of the sediment particles. $\varphi$ is the averaged porosity.

In classic SVE models the sign of $q_{b}$ coincides with the sign of $\tau$, the shear stress at the bottom. In general we can write

$$
\tau=\rho_{1} g \zeta\left(h_{1}\right) u_{1}\left|u_{1}\right|
$$

being $\zeta\left(h_{1}\right)$ a function depending on the considered friction law. The shear stress is usually defined as

$$
\tau=\rho_{1} g h_{1} S_{\mathrm{f}},
$$

being $S_{\mathrm{f}}$ the friction term. $S_{\mathrm{f}}$ can be described by different empirical laws, such as the Darcy-Weisbach $\left(S_{\mathrm{f}}=\right.$ $f u_{1}\left|u_{1}\right| / 8 g h_{1}$, where $f$ is the Darcy-Weisbach coefficient) or Manning formulae $\left(S_{\mathrm{f}}=n^{2} u_{1}\left|u_{1}\right| / h_{1}^{4 / 3}\right.$, where $n$ is the Manning coefficient). 
The Shields stress, $\theta$, represents the ratio between the agitating and the stabilizing forces on a sediment grain in the bed,

$$
\theta=\frac{|\tau| d_{s}^{2}}{g\left(\rho_{2}-\rho_{1}\right) d_{s}^{3}}
$$

and $\theta_{c}$ is the critical Shields stress for incipient motion.

The constants $k_{l}, m_{l}, l=1,2,3$ are positive real numbers. Depending on the values of theses constants, we obtain different models. Usually at least one of the parameters $m_{1}, m_{2}$ or $m_{3}$ is set to zero. For example, Meyer-Peter and Müller's model is defined by

$$
\frac{q_{b}}{Q}=\operatorname{sgn}(\tau) \frac{8}{(1-\varphi)}\left(\theta-\theta_{c}\right)_{+}^{3 / 2}
$$

and Ashida and Michiue's model is defined by:

$$
\frac{q_{b}}{Q}=\operatorname{sgn}(\tau) \frac{17}{(1-\varphi)}\left(\theta-\theta_{c}\right)_{+}\left(\sqrt{\theta}-\sqrt{\theta_{c}}\right) .
$$

Finally, by $(\cdot)_{+}$we denote the positive part. In equation (1.2) the positive part implies that the sediment moves when the modulus of the shear stress is bigger than a given critical value.

Although the classic SVE model is largely used, it presents several disadvantages:

(i) The SVE model has not a dissipative energy equation associated to the system.

(ii) Solid transport discharge formulae are derived by using the hypothesis of nearly horizontal sediment beds, that is, $\nabla_{x} \eta \approx 0$.

(iii) Solid transport flux is independent of the thickness of the sediment layer. Thus, the mass conservation property for the sediment given by third equation in (1.1) may fail (see [39]).

Concerning the first item, we should remark that there exist in the literature some simpler solid transport formulae for which the corresponding SVE model has an associated dissipative energy equation (see for example [60]). Nevertheless, up to our knowledge, no general result exists in the bibliography in this sense.

The second item implies that classic formulae cannot be used in several problems of interest (see [33]) because they are derived by using the hypothesis of nearly horizontal sediment beds.

As mentioned before, the Shields parameter is the ratio between agitating forces and the stabilizing forces. Classical formulae consider that the only agitating force is the bottom shear stress, concretely $|\tau| d_{s}^{2}$. Nevertheless, in the experiments presented by Lysne in [36] it can be seen that gravity is another contributing factor as an agitating force (see also [27]) for sloped sediment beds. Thus, it is necessary to take into account gravitational forces in order to obtain a solid transport discharge that can be applied for arbitrarily sloping beds.

This has been achieved in the literature in several ways. For instance, the simplest way to take into account the sediment bed slope in the definition of the solid transport discharge is to include a diffusion term. Engelund and Hansen proposed in [19] a formula that can be written under the following form,

$$
q_{b}=k\left|u_{1}\right|^{m}\left(\frac{u_{1}}{\left|u_{1}\right|}-c \nabla_{x} \eta\right),
$$

$k, m$, and $c$ being constant parameters of the model (see also [57,58]). Equation (1.7) can be seen as a modification of the Grass model. An adaptation of this formula for curved channels was proposed by Struiksma et al. in [56]:

$$
q_{b}=k\left|u_{1}\right|^{m}\left(1-c \partial_{s}\left(b+h_{2}\right)\right) \operatorname{sgn}\left(u_{1}-\frac{1}{f_{s} \theta} \nabla_{x} \eta\right)
$$

$s$ being the streamwise coordinate and $f_{s}$ the shape factor of the grains (see [56] for more details). Note that this definition implies that the direction of the sediment transport does not coincide with the direction of the velocity 
of the fluid. The direction is determinated by the sign of the vector $\left(u_{1}-\frac{1}{f_{s} \theta} \nabla_{x} \eta\right)$. Note that

$$
\operatorname{sgn}\left(u_{1}-\frac{1}{f_{s} \theta} \nabla_{x} \eta\right)=\frac{u_{1}-\frac{1}{f_{s} \theta} \nabla_{x} \eta}{\left|u_{1}-\frac{1}{f_{s} \theta} \nabla_{x} \eta\right|}=\left(\begin{array}{c}
\cos \alpha \\
\sin \alpha
\end{array}\right),
$$

$\alpha$ being the angle of the transport direction, where

$$
\tan \alpha=\frac{u_{1[2]}-\frac{1}{f_{s} \theta} \partial_{x_{2}} \eta}{u_{1[1]}-\frac{1}{f_{s} \theta} \partial_{x_{1}} \eta} .
$$

Let us remark that in [56] the direction of the sediment transport is defined in terms of its angle, given by (1.8), rather than defining it in terms of the sign vector. Authors also include a correction of the angle due to the transversal velocity in curves. For the sake of brevity we do not include here a discussion on the modification of the transport angle in curved channels, which is not the aim of this paper.

A more used extension of classic formulae for arbitrarily sloping sediment beds is to consider a modification of the critical Shields parameter, replacing $\theta_{c}$ by $\widehat{\theta}_{c}$ (see $[20,27]$ ). For the scalar case, $1 \mathrm{D}$ flows, this modification can be written as follows:

$$
\widehat{\theta}_{c}=\theta_{c}\left(1+\frac{\operatorname{sgn}(\tau)}{\tan \delta} \partial_{x} \eta\right)=\theta_{c}+\vartheta \operatorname{sgn}(\tau) \partial_{x} \eta, \quad \text { where } \quad \vartheta=\frac{\theta_{c}}{\tan \delta} .
$$

In the works of Kovacs and Parker [33], Seminara et al. [55] and Parker et al. [46] several extensions for the vectorial case are presented, where the computation of $\widehat{\theta}_{c}$ takes into account lateral slopes.

Note that the definition of the modified critical Shields parameter do not usually take into account the sign of $\tau$. This is due to the fact that formulae are usually presented for the case of positive velocities only.

In previous definition $\tan \delta$ is the friction coefficient corresponding to the internal friction angle of the material. Typical values of $\vartheta$ and $\theta_{c}$ are, $\vartheta=0.1$ and $\theta_{c}=0.047$, what implies that $\theta_{c} / \vartheta=\tan \delta$, with $\delta \approx 25^{\circ}$, as proposed by Fredsœe in [27]. The angle $\delta=25^{\circ}$ is lower than the usual repose angle of $32^{\circ}$, although lower values have also been suggested (see [9] and references therein).

Let us remark that the definition of $\widehat{\theta}_{c}$ in (1.9) is based on two ideas: first, gravitational forces due to the sediment bed slope are incorporated as agitating forces in the definition of the effective Shields parameter,

$$
\theta_{\mathrm{eff}}=\frac{\left|\tau_{\mathrm{eff}}\right| d_{s}^{2}}{g\left(\rho_{2}-\rho_{1}\right) d_{s}^{3}} \quad \text { with } \quad \tau_{\mathrm{eff}}=\tau-\vartheta\left(\rho_{2}-\rho_{1}\right) g d_{s} \partial_{x}\left(b+h_{2}\right),
$$

where $\tau_{\text {eff }}$ is the effective shear stress. Second, rather than replacing $\theta$ by $\theta_{\text {eff }}$, it is usually assumed that this is equivalent to replacing $\theta_{c}$ by $\widehat{\theta}_{c}$, defined by (1.9) (see [27]). More explicitly, it is usually assumed that $\left(\theta_{\text {eff }}-\theta_{c}\right)_{+}=\left(\theta-\widehat{\theta}_{c}\right)_{+}$.

Remark 1.1. Let us consider the simplest case, corresponding to $1 \mathrm{D}$ models, $u_{1}>0$ and $\partial_{x} \eta<0$. And let us suppose that $\partial_{x} \eta=-\left(\tan \delta+\tan \delta_{\epsilon}\right) \approx-\tan \left(\delta+\delta_{\epsilon}\right)$, with $\delta_{\epsilon} \geq 0$. Then, we obtain

$$
\left(\theta_{\text {eff }}-\theta_{c}\right)_{+}=\left(\theta-\hat{\theta}_{c}\right)_{+}=\left(\theta+\vartheta \tan \delta_{\epsilon}\right)_{+} .
$$

As as consequence, if $\delta_{\epsilon}=0$, and if the fluid moves, that is, if $\theta>0$, then the sediment also moves. Because the slope of the sediment layer in this case coincides with the repose angle of the material. Then, the material is at rest if there is not any extra force. And it moves if there is friction with the water.

We can also observe that if $\delta_{\epsilon}>0$ then the sediment moves (even if the fluid is at rest, that is, $\theta=0$ ). Because if $\delta_{\epsilon}>0$ then the slope of the sediment layer is bigger than the repose angle. 
Nevertheless, it is not true in general that $\left(\theta_{\mathrm{eff}}-\theta_{c}\right)_{+}=\left(\theta-\widehat{\theta}_{c}\right)_{+}$, because

$$
\theta_{\mathrm{eff}}-\theta_{c} \neq \frac{|\tau| d_{s}^{2}-\vartheta \operatorname{sgn}(\tau) g\left(\rho_{2}-\rho_{1}\right) d_{s}^{3} \partial_{x}\left(b+h_{2}\right)}{g\left(\rho_{2}-\rho_{1}\right) d_{s}^{3}}-\theta_{c}=\theta-\widehat{\theta}_{c} .
$$

The problem arises from the fact that the absolute value (or the norm in the vectorial case) is neglected in the definition of $\theta_{\text {eff }}$ (see for example $[9,27]$ ), which should be taken into account. In other words, in general situations the sign of $\tau$ and $\tau_{\text {eff }}$ should be considered.

In fact, Fowler et al. proposed in [26] a modification of the Meyer-Peter and Müller formula that consist in replacing $\theta$ by $\theta_{\text {eff }}$, instead of replacing $\theta_{c}$ by $\widehat{\theta}_{c}$. The model proposed by Fowler et al. can be written as follows:

$$
\frac{q_{b}}{Q}=8 \operatorname{sgn}\left(\tau_{\mathrm{eff}}\right) \frac{h_{2}}{\bar{h}_{2}}\left(\theta_{\mathrm{eff}}-\theta_{c}\right)_{+}^{3 / 2},
$$

where $\bar{h}_{2}$ is an averaged value of the thickness of the sediment layer. The fact of introducing an explicit dependence on $h_{2}$ in the formula is also interesting. Indeed, this allows to ensure the mass conservation property for the sediment layer (see also [39]), which was the second issue noted previously as a disadvantage for classic SVE models.

Related to this problem, in [24], Fernández-Nieto et al. introduce a modified general definition of the solid transport discharge for SVE models that takes into account the thickness of the sediment layer. Then mass conservation is ensured. Moreover, the proposed formula has the advantage that it reduces to a classic solid transport discharge formula in the case of quasi-uniform regimes. This is in fact the regime where classic formulae are usually derived.

The main objective of this paper is to show that the SVE model can be deduced through an asymptotic analysis of the Navier-Stokes equations. Moreover, we obtain that following this process some improvements on the classic SVE model are achieved: (i) we get that the deduced model verifies exactly a dissipative energy equation. This deduction and the proof of energy allows us to understand why classic models do not have an associated energy. Moreover, it allows to prove that the classic SVE model has an associated energy by introducing a simple modification. (ii) In the deduction process of the model the pressure terms introduce a modification that should be taken into account if we consider applications where the sediment bed is not nearly horizontal. We also see that this modification coincides with some of the alternatives proposed in the literature for some special cases. (iii) The solid transport flux depends on the thickness of the moving sediment layer, then mass conservation is ensured.

The paper is organized as follows: in Section 2 we present the 3D system of equations considered as starting point from which the models are deduced, the proposed models and the results on the associated energy. The formal deduction of the models by an asymptotic analysis from the Navier-Stokes equations is detailed in Appendix A. This is done by developing a multi-scale analysis in space and time. The proofs of the energy balance results (Thms. 2.4 and 2.6) are presented in Appendix B. Section 3 is devoted to numerical tests. Finally, the conclusions are presented in Section 4.

\section{The ASymptotic SAint-Venant-Exner Models}

Following an asymptotic analysis, we derive two main models which are presented here (see Appendix A for details). The difference between these models is the considered friction law at the interface between the fluid and sediment. In Section 2.1 we summarize the starting 3D system of equations and the hypothesis considered for the derivation of the models. The models deduced in this work are shown in Section 2.2. First, they are presented with the same notation as they are deduced (see Appendix A). Secondly, they are rewritten in terms of the Shields parameter or the effective Shields parameter. The associated energy for these models is presented in Section 2.3. Moreover, we include in Section 2.4 a result that justifies that classic SVE model may have an associated dissipative energy provided with a correction in the friction term appearing in the momentum equation. 


\subsection{The $3 \mathrm{D}$ initial system}

We consider two immiscible layers of different materials with different physical properties: velocity, pressure, density and viscosity. The two layers are related through the interaction terms at the internal interface levels. In the following subsections the starting systems of equations, the definition of the physical domain and the considered hypothesis for the deduction of the models proposed in this paper are detailed.

\subsubsection{Physical domain and governing equations}

We consider a cartesian coordinate system where $x$ represents the horizontal 2D direction and $z$ the vertical one. Let us define the physical domain for the fluid and sediment layers by $\Omega_{1}(t)$ and $\Omega_{2}(t)$ respectively; $t$ being the time variable. Usually in the context of bedload transport it is assumed that the sediment domain can be decomposed into two layers: one that moves due to the action of the convection of the upper fluid, the mobile sediment layer with thickness $h_{m}$, and a second one composed of sediment that is not moving but is susceptible to come into motion, with thickness $h_{f}$. These two layers are assumed to lay on a fixed bedrock layer that is not affected by the fluid. This leads us to define four boundaries in the domain (see Fig. 1):

$$
\begin{aligned}
\Gamma_{s} & =\left\{(x, z) \in \mathbb{R}^{3} / x \in \omega, z=\eta_{s}(x, t)\right\} \\
\Gamma_{1,2} & =\left\{(x, z) \in \mathbb{R}^{3} / x \in \omega, z=\eta(x, t)\right\} \\
\Gamma_{f} & =\left\{(x, z) \in \mathbb{R}^{3} / x \in \omega, z=\eta_{f}(x, t)\right\} \\
\Gamma_{b} & =\left\{(x, z) \in \mathbb{R}^{3} / x \in \omega, z=b(x)\right\} .
\end{aligned}
$$

where $\omega$ is a domain in $\mathbb{R}^{2}$. The water free surface is defined by $z=\eta_{s}(x, t)$, where $\eta_{s}(x, t)=h_{1}(x, t)+h_{2}(x, t)+$ $b(x)$ and the fluid/sediment interface by $z=\eta(x, t)$, where $\eta(x, t)=b(x)+h_{2}(x, t) . h_{1}(x, t)$ denotes the thickness of the water column. The sediment layer is decomposed as $h_{2}(x, t)=h_{m}(x, t)+h_{f}(x, t)$, and then the internal sediment interface is $z=\eta_{f}(x, t)$, where $\eta_{f}(x, t)=b(x)+h_{f}(x, t)$ (see Fig. 1).

Thus, we consider a time-dependant domain $\Omega(t)=\Omega_{1}(t) \cup \Omega_{2}(t) \cup \Gamma_{b} \cup \Gamma_{1,2}(t) \cup \Gamma_{s}(t)$, being:

$$
\begin{aligned}
& \Omega_{1}(t)=\left\{(x, z) \in \mathbb{R}^{3} / x \in \omega, \eta(x, t)<z<\eta_{s}(x, t)\right\} ; \\
& \Omega_{2}(t)=\Omega_{2, f}(t) \cup \Omega_{2, m}(t) ;
\end{aligned}
$$

where

$$
\begin{aligned}
\Omega_{2, f}(t) & =\left\{(x, z) \in \mathbb{R}^{3} / x \in \omega, b(x)<z<\eta_{f}(x, t)\right\} ; \\
\Omega_{2, m}(t) & =\left\{(x, z) \in \mathbb{R}^{3} / x \in \omega, \eta_{f}(x, t)<z<\eta(x, t)\right\} .
\end{aligned}
$$

Moreover, let us denote by $T_{m}$ the mass transference between the static and the mobile sediment domains, $\Omega_{2, f}$ and $\Omega_{2, m} . T_{m}$ is defined as the difference between the erosion rate $\left(\dot{z}_{e}\right)$ and the deposition rate $\left(\dot{z}_{d}\right), T_{m}=\dot{z}_{e}-\dot{z}_{d}$ (see [24] and Sect. 2.2.3).

As a general rule in notation, we will use the subscript 1 to denote the upper layer (fluid) and the subscript 2 for the lower layer (sediment). We denote by

$$
v_{i}=\left(u_{i}, w_{i}\right)
$$

the velocity field for each layer with $u_{i}=\left(u_{i[1]}, u_{i[2]}\right)$ the $2 \mathrm{D}$ horizontal velocity. We denote by $\rho_{i}$ the density and $p_{i}$ the pressure. Moreover, $\mu_{i}$ and $\nu_{i}=\mu_{i} / \rho_{i}$, denote the dynamic and kinematic viscosity coefficients respectively, for $i=1,2$.

For each layer $(i=1,2)$, we start from the 3D Navier-Stokes equations for incompressible fluid and sediment components:

$$
\left\{\begin{array}{l}
\rho_{i} \partial_{t} v_{i}+\left(\rho_{i} v_{i} \nabla\right) v_{i}-\operatorname{div}\left(\sigma_{i}\right)=-\rho_{i} \boldsymbol{g} \\
\operatorname{div}\left(v_{i}\right)=0
\end{array}\right.
$$

In this system $\boldsymbol{g}$ represents the gravitational vector and $\sigma_{i}$ the stress tensor associated to each layer. 


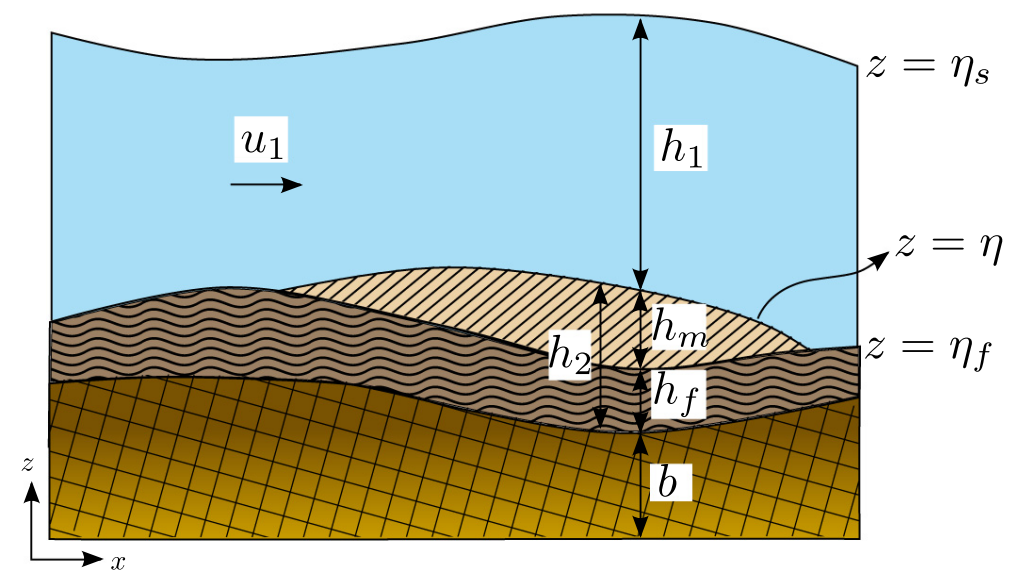

Figure 1. Sketch of the domain for the fluid-sediment problem.

To complete this system, we must set the stress tensor definition, the interactions at the internal interface levels $\left(\Gamma_{1,2}\right.$ and $\left.\Gamma_{f}\right)$, as well as boundary and kinematic conditions. This will be done in the following section.

Note that in the limit case when the whole sediment layer becomes static, $h_{m}=0$, then $\Gamma_{f}=\Gamma_{1,2}$. And when the whole sediment layer is moving, $h_{f}=0$, then $\Gamma_{f}=\Gamma_{b}$.

\subsubsection{Closures}

\section{Stress tensors}

We shall define the stress tensors as follows:

$$
\sigma_{i}=\sigma_{i}^{\prime}-p_{i} \mathrm{Id}, \quad \text { for } i=1,2 ;
$$

where $\sigma_{i}^{\prime}$ is the deviatoric part of the stress tensor.

We assume that the stress tensor for the fluid layer follows a Newtonian form with constant dynamic viscosity $\mu_{1}$. Then $\sigma_{1}^{\prime}$ is given by:

$$
\sigma_{1}^{\prime}=2 \mu_{1} D\left(v_{1}\right)
$$

$D(v)$ being the rate of deformation tensor, $D(v)=\frac{1}{2}\left(\nabla v+\nabla^{t} v\right)$.

For the sediment layer we consider a non-Newtonian rheology. Recent works have been devoted to demonstrate through experimental results the resemblance between the bedload transport phenomena and the granular flows behavior $[2,11,44,45]$. Ouriemi et al. proposed in [44] a two-phase model for bedload transport in laminar flows. Where a Newtonian law for the fluid phase and a frictional rheology for the particulate phase, namely a Coulomb type friction is considered. Chauchat et al. (see [7,49]) study a three-dimensional two-phase model where a Drucker-Prager rheology describe the stress tensor associated to the particulate phase. This definition is consistent with the Coulomb friction model proposed in [44]. A comparison with experimental data for bedload transport can be seen in [2].

Following these references we define

$$
\sigma_{2}^{\prime}=2 \mu_{2} D\left(v_{2}\right)
$$

with a non-constant viscosity $\mu_{2}$, given by a Drucker-Prager model. By using a regularization of the DruckerPrager model (see [35]), we can assume that $\mu_{2}$ is expressed as a function of $D\left(v_{2}\right)$ and $p_{2}$ :

$$
\mu_{2}=\mu_{2}\left(D\left(u_{2}\right), p_{2}\right) .
$$

Nevertheless, let us remark that in order to deduce a first order Reynolds-type model for the sediment layer, we do not need further specifications about this rheology. In fact, the depth-averaged model will be written 
in terms of the boundary conditions, that is, the Coulomb friction law, which is compatible with the DruckerPrager rheology.

\section{Friction laws}

We must define the friction laws at the mobile-static sediment interface and at the fluid-sediment interface.

- Friction law at the internal mobile-static sediment interface

As explained before, a Coulomb friction condition at the interface between the static and the moving sediment particles, $z=\eta_{f}(x, t)$, is considered. Denoting by $N_{f}$ the unitary normal vector to the interface $\Gamma_{f}$ and by $\delta$ the repose angle, we write the Coulomb friction law as:

$$
\left(\sigma_{2} N_{f}\right)_{\mathcal{T}}=-\left(\operatorname{sgn}\left(u_{2}\right) \tan \delta\left(\left(\sigma_{1}-\sigma_{2}\right) N_{f}\right) N_{f}\right)_{\mid z=\eta_{f}},
$$

where $N_{f}=\left(-\nabla_{x} \eta_{f}, 1\right)^{t} / \sqrt{1+\left|\nabla_{x} \eta_{f}\right|^{2}}$ and the subscript $(\cdot)_{\mathcal{T}}$ denotes the tangent component of a vector.

Let us remark that the use of a Coulomb friction can also be interpreted as a mechanism to approximate the collision effects of saltating grains in the computation of the bedload transport formula (see [33]).

- Friction law at the fluid-sediment interface

At the fluid-sediment interface the interaction between fluid and sediment is defined through a friction force:

$$
\left(\sigma_{1} N_{\eta}\right)_{\mathcal{T}}=\left(\sigma_{2} N_{\eta}\right)_{\mathcal{T}}=\text { fric, }
$$

where $N_{\eta}=\left(-\nabla_{x} \eta, 1\right)^{t} / \sqrt{1+\left|\nabla_{x} \eta\right|^{2}}$ is the normal vector at the interface pointing from layer 2 to layer 1 .

In particular, we consider two classic friction laws for which we obtain two different models that can be written under the same structure.

- Linear friction law:

$$
\text { fric }=C\left(u_{1}-u_{2}\right)_{\mid z=\eta}
$$

where the coefficient $C$ has velocity dimension. Moreover, taking into account the results presented in [41] (see also Rem. A.1), $C$ can be assumed proportional to $h_{m}$. Following the analysis of Seminara et al. [55], the drag coefficient is proportional to $\tan (\delta) / \theta_{c}$. That is, inversely proportional to $\vartheta$, defined in (1.9). Taking into account these remarks we may define

$$
C=\frac{(1 / r-1) g h_{m}}{\vartheta \sqrt{(1 / r-1) g d_{s}}}
$$

- Quadratic friction law:

$$
\text { fric }=C_{1}\left|\left(u_{1}-u_{2}\right)_{\mid z=\eta}\right|\left(u_{1}-u_{2}\right)_{\mid z=\eta} .
$$

In this case $C_{1}$ must be adimensional, so taking into account previous arguments on the definition of the drag coefficient, we can define

$$
C_{1}=\frac{h_{m}}{\vartheta d_{s}}
$$

\section{Boundary and kinematic conditions}

The following boundary and kinematic conditions are imposed at each interface to complete the system:

- At the free surface, $z=\eta_{s}(t, x)=b(x)+h_{2}(x, t)+h_{1}(x, t)$ :

- The surface tension condition: $\sigma_{1} N_{s}=0$ where $N_{s}=\frac{1}{\sqrt{1+\left|\nabla_{x} \eta_{s}\right|^{2}}}\left(-\nabla_{x} \eta_{s}, 1\right)^{t}$ is the unitary outward normal vector to the free surface and the subscript $n$ denotes the normal component.

- The kinematic condition: $\partial_{t} \eta_{s}=v_{1} \cdot N_{s}$. 
- At the fluid/sediment interface, $z=\eta(t, x)=b(x)+h_{2}(x, t)$ :

- The kinematic conditions corresponding to both velocities:

$$
\partial_{t} \eta=v_{1} \cdot N_{\eta}=v_{2} \cdot N_{\eta}
$$

- The continuity of the normal component of the tensors: $\left(\sigma_{1} \cdot N_{\eta}\right)_{n}=\left(\sigma_{2} \cdot N_{\eta}\right)_{n}$.

- The friction law defined by (2.3)-(2.7).

- At the internal sediment interface, $z=\eta_{f}(t, x)=b(x)+h_{f}(x, t)$ :

- The conservation of the sediment mass,

$$
\partial_{t} \eta_{f}=v_{2} \cdot N_{f}-T_{m}
$$

$T_{m}$ being the mass transference term (see Sect. 2.2.3).

- The Coulomb friction law defined by $(2.2)$.

- At the bottom, $z=b(x)$ :

- The no penetration condition: $v_{2} \cdot N_{b}=0$, where the unitary normal vector to the bottom is $N_{b}=$ $\left(-\nabla_{x} b, 1\right)^{t} / \sqrt{1+\left|\nabla_{x} b\right|^{2}}$.

\subsection{Proposed models}

In this section we present the final models obtained through an asymptotic analysis of the 3D system (2.1). Following the work performed in [23], we derive a mathematical two dimensional SVE type model for bedload transport. Thus, the models are composed by three equations: the first two equations represent the Saint-Venant system that describe the upper fluid layer and the third one corresponds to the evolution of the moving bed through a lubrication Reynolds equation.

As it is classically considered in such kind of models, we take into account two different time scales for the hydrodynamics and the sediment evolution. Therefore, the ratio between the hydrodynamic and morphodynamic time scales, defined by $\varepsilon^{2}$, being small. We obtain first order models, so we neglect the terms of order $\varepsilon^{2}$, as commonly done, to obtain SVE type models, (see [28] and references therein).

The two obtained models depend on the considered friction law at the fluid-sediment interface, given by $(2.4)$ and (2.6) respectively. The complete derivation of these models is detailed in Appendix A.

First, we present the models deduced under a unified formulation. Then, we analyze each of them in order to relate the sediment velocity with the classic motion threshold in terms of the Shields parameter and the effective Shields parameter. Finally, the closure of the systems is discussed and simplified models corresponding to a quasi stationary regime will be presented. From now on, we use the superscript $(L F)$ to denote the properties of the model deduced with a linear friction law, and $(Q F)$ for the model using a quadratic friction law.

The model deduced in this paper can be written under the form of a SVE system form as follows:

$$
\left\{\begin{array}{l}
\partial_{t} h_{1}+\operatorname{div}_{x} q_{1}=0, \\
\partial_{t} q_{1}+\operatorname{div}_{x}\left(h_{1}\left(u_{1} \otimes u_{1}\right)\right)+\frac{1}{2} g \nabla_{x} h_{1}^{2}+g h_{1} \nabla_{x}\left(b+h_{2}\right)+\frac{g h_{m}}{r} \mathcal{P}=0, \\
\partial_{t} h_{2}+\operatorname{div}_{x}\left(h_{m} v_{b} \sqrt{(1 / r-1) g d_{s}}\right)=0, \\
\partial_{t} h_{f}=-T_{m} .
\end{array}\right.
$$

with

$$
\mathcal{P}=\nabla_{x}\left(r h_{1}+h_{2}+b\right)+(1-r) \operatorname{sgn}\left(u_{2}\right) \tan \delta .
$$


and the velocity $v_{b}$ defined for each friction law as:

$$
\begin{aligned}
v_{b}^{(L F)} & =\frac{1}{\sqrt{(1 / r-1) g d_{s}}} u_{1}-\frac{\vartheta}{1-r} \mathcal{P}, \\
v_{b}^{(Q F)} & =\frac{1}{\sqrt{(1 / r-1) g d_{s}}} u_{1}-\left(\frac{\vartheta}{1-r}\right)^{1 / 2}|\mathcal{P}|^{1 / 2} \operatorname{sgn}(\mathcal{P}) .
\end{aligned}
$$

Note that the term $\mathcal{P}$ is a modification of the velocity of the sediment $v_{b}$. The first addend in $\mathcal{P}$ introduces the gravitational effects in the sediment discharge coming from the pressure action. The second addend comes from the Coulomb friction law at the internal sediment boundary. Let us remark that $\operatorname{sgn}\left(u_{2}\right)$ has to be defined yet, for the convenience of the explanation, it is given below in equation (2.13)

We can write these velocities in terms of the Shields parameter. With this purpose, we use now the expression of the shear stress and the definitions of the friction coefficients $C$ and $C_{1}$ given by (2.5) and (2.7). We obtain,

$$
v_{b}^{(L F)}=\operatorname{sgn}\left(\tau_{\mathrm{eff}}^{(L F)}\right)\left(\theta_{\mathrm{eff}}^{(L F)}-\theta_{c}\right)_{+},
$$

where $\tau_{\text {eff }}^{(L F)}$ and $\theta_{\text {eff }}^{(L F)}$ are defined by (2.15) and (2.16), respectively, for the case of a linear friction law. For the case of a quadratic friction law $v_{b}^{(Q F)}$ is defined by (2.26). If we consider a linearization of the quadratic friction law (see Subsect. 2.2.2), we obtain

$$
v_{b}^{(Q F)}=\operatorname{sgn}\left(\tau_{\text {eff }}^{(Q F)}\right)\left(\left(\theta_{\text {eff }}^{(Q F)}\right)^{1 / 2}-\theta_{c}^{1 / 2}\right)_{+},
$$

where $\tau_{\text {eff }}^{(Q F)}$ and $\theta_{\text {eff }}^{(Q F)}$ are defined by (2.23)-(2.24).

In the following subsections we describe the deduction of previous definitions of $v_{b}$ for each friction law.

\subsubsection{Model deduced with the linear friction law (2.4) and (2.5)}

From the asymptotic approximation at first order, we introduce the shear stress and the Shields parameter as

$$
\frac{\tau^{(L F)}}{\rho_{1}}=C u_{1} \quad \text { and } \quad \theta^{(L F)}=\frac{\left|\tau^{(L F)}\right| / \rho_{1}}{(1 / r-1) g d_{s}}=\frac{C\left|u_{1}\right|}{(1 / r-1) g d_{s}} .
$$

Using the definition of the friction coefficient $C$ in equation (2.5), we have

$$
\frac{u_{1}}{\sqrt{(1 / r-1) g d_{s}}}=\vartheta \operatorname{sgn}\left(u_{1}\right) \frac{d_{s}}{h_{m}} \theta^{(L F)}
$$

Thus, taking into account (2.9) and the definition of $\vartheta$ in (1.9), the velocity of the sediment layer reads in this case

$$
v_{b}^{(L F)}=\vartheta \operatorname{sgn}\left(u_{1}\right) \frac{d_{s}}{h_{m}} \theta^{(L F)}-\frac{\vartheta}{1-r} \nabla_{x}\left(r h_{1}+\eta\right)-\operatorname{sgn}\left(u_{2}\right) \theta_{c} .
$$

- Influence of the Coulomb friction law.

Note that the sign of the velocity of the sediment layer, $\operatorname{sgn}\left(u_{2}\right)$, has still to be defined. Observe that this coefficient comes from the contribution of the Coulomb friction law at the interface between moving and static sediment particles (see (2.9)). In order to specify the sign of $u_{2}$, we remark that Coulomb friction force has the same sign as the net force acting on the sediment. That is,

$$
\operatorname{sgn}\left(\frac{\vartheta h_{m}}{1-r}(1-r) \operatorname{sgn}\left(u_{2}\right) \tan \delta\right)=\operatorname{sgn}\left(\frac{h_{m} u_{1}}{\sqrt{(1 / r-1) g d_{s}}}-\frac{\vartheta h_{m}}{1-r} \nabla_{x}\left(r h_{1}+h_{2}+b\right)\right) .
$$


Then, using that $\vartheta=\frac{\theta_{c}}{\tan \delta}$,

$$
\operatorname{sgn}\left(u_{2}\right)=\operatorname{sgn}\left(\frac{u_{1}}{\sqrt{(1 / r-1) g d_{s}}}-\frac{\vartheta}{1-r} \nabla_{x}\left(r h_{1}+h_{2}+b\right)\right) .
$$

Note also that this relation can be directly obtained from $(2.12)$. As $\operatorname{sgn}\left(u_{2}\right)=\operatorname{sgn}\left(v_{b}^{(L F)}\right)$, then from $(2.12)$ we deduce:

$$
\operatorname{sgn}\left(u_{2}\right)\left(\left|v_{b}^{(L F)}\right|+\vartheta \tan \delta\right)=\frac{u_{1}}{\sqrt{(1 / r-1) g d_{s}}}-\frac{\vartheta}{1-r} \nabla_{x}\left(r h_{1}+h_{2}+b\right)
$$

And as a consequence we obtain (2.13).

Coulomb friction force also introduces a threshold. It implies that the velocity of sediment layer is zero when the net forces acting on the sediment is not large enough to compensate the friction force. In this case, we obtain that the velocity of the sediment layer $v_{b}$ must be zero under the following condition:

$$
\left|\frac{h_{m} u_{1}}{\sqrt{(1 / r-1) g d_{s}}}-\frac{\vartheta h_{m}}{1-r} \nabla_{x}\left(r h_{1}+h_{2}+b\right)\right| \leq \frac{\vartheta h_{m}}{1-r}(1-r) \tan \delta=h_{m} \theta_{c} .
$$

\section{- Link with the effective and the critical Shields parameter.}

In order to take into account the stop criterion (2.14), we introduce the modified shear stress including the gravitational forces, called the "effective shear stress". It is defined in this case as:

$$
\frac{\tau_{\mathrm{eff}}^{(L F)}}{\rho_{1}}=\frac{\vartheta d_{s}}{h_{m}} \frac{\tau^{(L F)}}{\rho_{1}}-\frac{\vartheta g d_{s}}{r} \nabla_{x}\left(r h_{1}+h_{2}+b\right)
$$

The corresponding definition of the effective Shields parameter is

$$
\theta_{\mathrm{eff}}^{(L F)}=\frac{\left|\tau_{\mathrm{eff}}^{(L F)}\right| / \rho_{1}}{(1 / r-1) g d_{s}}=\frac{\operatorname{sgn}\left(\tau_{\mathrm{eff}}^{(L F)}\right)}{(1 / r-1) g d_{s}}\left(\vartheta \operatorname{sgn}\left(u_{1}\right) \frac{d_{s}}{h_{m}} \theta^{(L F)}-\frac{\vartheta}{1-r} \nabla_{x}\left(r h_{1}+h_{2}+b\right)\right) .
$$

In particular, with these definitions we obtain that $\operatorname{sgn}\left(\tau_{\mathrm{eff}}^{(L F)}\right)=\operatorname{sgn}\left(u_{2}\right)$ thanks to (2.13) and the definition of $\tau^{(L F)}$. As a consequence, from (2.12), the velocity of the sediment layer can be written as:

$$
v_{b}^{(L F)}=\operatorname{sgn}\left(\tau_{\mathrm{eff}}^{(L F)}\right)\left(\theta_{\mathrm{eff}}^{(L F)}-\theta_{c}\right)_{+} .
$$

Observe that condition (2.14) coming from the Coulomb friction law is equivalent to the classic one, $\theta_{\text {eff }}^{(L F)}>\theta_{c}$.

Remark 2.1 (Comparison with the classic effective shear stress). The definition of the effective shear stress (2.15) can be seen as a generalization of the classic one (1.10). It can be written in vectorial form as follows (see [26]),

$$
\frac{\tau_{\mathrm{eff}}}{\rho_{1}}=\frac{\tau}{\rho_{1}}-\vartheta(1 / r-1) g d_{s} \nabla_{x}\left(h_{2}+b\right)
$$

When comparing the two expressions we find two main differences: the first one is the factor $\left(\vartheta d_{s} / h_{m}\right)$ multiplying the shear stress, which comes from the fact that the model is expressed in terms of the thickness of the moving sediment layer. The second one appears in the second term, which is given by $\left(g d_{s} \vartheta \nabla_{x}\left(r h_{1}+h_{2}+b\right) / r\right)$ instead of $\left(g d_{s} \vartheta(1 / r-1) \nabla_{x}\left(h_{2}+b\right)\right)$. Both terms are related:

$$
\begin{aligned}
g d_{s} \vartheta(1 / r-1) \nabla_{x}\left(h_{2}+b\right) & =\frac{g d_{s} \vartheta}{r}\left(\nabla_{x}\left(r h_{1}+h_{2}+b\right)-r \nabla_{x}\left(h_{1}+h_{2}+b\right)\right) \\
& =\frac{g d_{s} \vartheta}{r} \nabla_{x}\left(r h_{1}+h_{2}+b\right)-g d_{s} \vartheta \nabla_{x}\left(h_{1}+h_{2}+b\right) .
\end{aligned}
$$

So that both definitions coincide in the case of constant free surface, $\nabla_{x}\left(h_{1}+h_{2}+b\right)=0$. Nevertheless, this additional term $g d_{s} \vartheta \nabla_{x}\left(h_{1}+h_{2}+b\right)$ could be relevant in more general situations. 


\subsubsection{Model deduced with the quadratic friction law (2.6)-(2.7)}

For the quadratic friction law we have the following definition of the fluid shear stress for the first order approximation

$$
\frac{\tau^{(Q F)}}{\rho_{1}}=C_{1}\left|u_{1}\right| u_{1}, \quad \text { and then } \quad \theta^{(Q F)}=\frac{C_{1}\left|u_{1}\right|^{2}}{(1 / r-1) g d_{s}} .
$$

Then, using the definition of $C_{1}$ in (2.7) and again (2.9) and (1.9), the velocity of the sediment is written as

$$
\begin{aligned}
v_{b}^{(Q F)}= & \operatorname{sgn}\left(u_{1}\right)\left(\vartheta \frac{d_{s}}{h_{m}} \theta^{(Q F)}\right)^{1 / 2} \\
& -\left|\frac{\vartheta}{1-r} \nabla_{x}\left(r h_{1}+h_{2}+b\right)+\operatorname{sgn}\left(u_{2}\right) \theta_{c}\right|^{1 / 2} \operatorname{sgn}\left(\frac{\vartheta}{1-r} \nabla_{x}\left(r h_{1}+h_{2}+b\right)+\operatorname{sgn}\left(u_{2}\right) \theta_{c}\right) .
\end{aligned}
$$

Following analogous arguments as in the case of linear friction law, we can define

$$
\operatorname{sgn}\left(u_{2}\right)=\operatorname{sgn}(\Phi)
$$

where

$$
\Phi=\operatorname{sgn}\left(u_{1}\right)\left(\vartheta \frac{d_{s}}{h_{m}} \theta^{(Q F)}\right)^{1 / 2}-\left|\frac{\vartheta}{1-r} \nabla_{x}\left(r h_{1}+h_{2}+b\right)\right|^{1 / 2} \operatorname{sgn}\left(\frac{\vartheta}{1-r} \nabla_{x}\left(r h_{1}+h_{2}+b\right)\right),
$$

and similarly to (2.14), the stop criterion is

$$
\left|h_{m} \Phi\right| \leq h_{m} \theta_{c} .
$$

For this model we introduce the effective shear stress as

$$
\tau_{\text {eff }}^{(Q F)}=g(1 / r-1) d_{s}|\Phi| \Phi \quad \text { and } \quad \theta_{\text {eff }}^{(Q F)}=|\Phi|^{2},
$$

where $\Phi$ is defined by (2.23). The former stop criterion, derived directly from the model with the Coulomb friction law, becomes

$$
\theta_{\mathrm{eff}}^{(Q F)}>\theta_{c}
$$

Observe that in this case we are not able to obtain an explicit expression of the velocity $v_{b}^{(Q F)}$ in terms of $\theta_{\text {eff }}^{(Q F)}$, due to the presence of the modulus and the sign vectors. Nevertheless, the definition of $v_{b}^{(Q F)}$ must take into account previous criterion. Thus, thanks to (2.21) and (2.25), we give

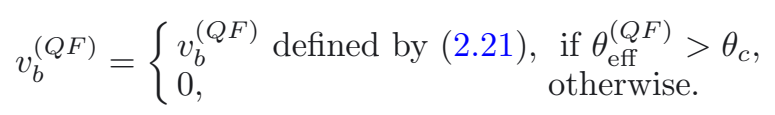

We may also consider the following approximation, which can be seen as a linearization, of the quadratic friction law (2.6):

$$
\text { fric }=C_{1}\left|\left(u_{1}-u_{2}^{*}\right)_{\mid z=\eta}\right|\left(u_{1}-u_{2}^{*}\right)_{\mid z=\eta}+C_{1}\left|\left(u_{2}^{*}-u_{2}\right)_{\mid z=\eta}\right|\left(u_{2}^{*}-u_{2}\right)_{\mid z=\eta},
$$

being $u_{2}^{*}$ the velocity of the system without considering the Coulomb friction law at the internal interface of the sediment layer. In this case, we obtain the following bedload velocity:

$$
v_{b}^{(Q F)}=\operatorname{sgn}\left(\tau_{\text {eff }}^{(Q F)}\right)\left(\left(\theta_{\text {eff }}^{(Q F)}\right)^{1 / 2}-\theta_{c}^{1 / 2}\right)_{+} .
$$

This corresponds to defining a solid transport discharge with a structure similar to that of classic formulae (see for example the Ashida and Michiue's model (1.6)).

Remark 2.2. Definition (2.24) of the effective shear stress is different from the classic ones. Nevertheless, as in previous case (see Eq. (2.15)), the main difference is to consider the term $\nabla_{x}\left(r h_{1}+h_{2}+b\right) /(1-r)$ instead of $\nabla_{x}\left(h_{2}+b\right)$. Note also that a linearization of (2.24) leads to previous definition of the effective shear stress $(2.15)$. 


\subsubsection{Sediment mass transference and simplified models}

To close the models, it is necessary to define $T_{m}$, the mass transference between the moving and the static sediment layers. It is defined in terms of the difference between the erosion rate, $\dot{z}_{e}$, and the deposition rate $\dot{z}_{d}$,

$$
T_{m}=\dot{z}_{e}-\dot{z}_{d}
$$

Following the definitions used in [24],

$$
\dot{z}_{e}=K_{e} \frac{Q}{d_{s}(1-\varphi)}\left(\theta-\theta_{c}\right)_{+}, \quad \dot{z}_{d}=K_{d} Q \frac{h_{m}}{d_{s}^{2}},
$$

$K_{e}$ and $K_{d}$ being the erosion and deposition constants respectively.

Nevertheless, it is necessary to remark that in such definition, the erosion rate does not take into account gravitational effects appearing in arbitrarily sloping beds. Thus, for the case of sediment beds which are not nearly horizontal, we propose to replace $\theta$ by $\theta_{\text {eff }}$. Thus, we set

$$
\dot{z}_{e}=K_{e} \frac{Q}{d_{s}(1-\varphi)}\left(\theta_{\mathrm{eff}}-\theta_{c}\right)_{+}
$$

with $\theta_{\text {eff }}$ defined by $(2.16)$ or $(2.24)$.

A simplification of the proposed model is to consider a quasi-uniform regime, where the deposition rate equals the erosion rate, that is $T_{m}=\dot{z}_{e}-\dot{z}_{d}=0$. In this case, from the definition of $\dot{z}_{d}$ and $\dot{z}_{e}$, we have

$$
h_{m}=\frac{K_{e} d_{s}}{K_{d}(1-\varphi)}\left(\theta_{\mathrm{eff}}-\theta_{c}\right)_{+} .
$$

This definition of the thickness of the moving sediment layer is consistent with the classic one deduced by Bagnold (see $[3,24]$ ), and with the experiments of Fernández Luque and van Beek (see $[9,20])$. Which states that $h_{m}$ is proportional to $\left(\theta-\theta_{c}\right)_{+}$.

If we introduce this definition of $h_{m}$ in (2.8), we obtain the following system

$$
\left\{\begin{array}{l}
\partial_{t} h_{1}+\operatorname{div}_{x} q_{1}=0 \\
\partial_{t} q_{1}+\operatorname{div}_{x}\left(h_{1}\left(u_{1} \otimes u_{1}\right)\right)+\frac{1}{2} g \nabla_{x} h_{1}^{2}+g h_{1} \nabla_{x}\left(b+h_{2}\right)+\frac{g h_{m}}{r} \mathcal{P}=0, \\
\partial_{t} h_{2}+\operatorname{div}_{x}\left(\frac{K_{e} d_{s}}{K_{d}(1-\varphi)}\left(\theta_{\mathrm{eff}}-\theta_{c}\right)_{+} v_{b} \sqrt{(1 / r-1) g d_{s}}\right)=0
\end{array}\right.
$$

with $v_{b}$ and $\theta_{\text {eff }}$ defined by $(2.15)-(2.17)$ for $(L F)$ and by $(2.21)-(2.24)$ for $(Q F)$.

Let us focus on the case of a linearization of the quadratic friction law, for which $v_{b}$ is defined by $(2.27)$. Then, we obtain a SVE model with the following definition of the solid transport discharge,

$$
\frac{q_{b}}{Q}=\operatorname{sgn}\left(\tau_{\mathrm{eff}}\right) \frac{K_{e}}{K_{d}(1-\varphi)}\left(\theta_{\mathrm{eff}}-\theta_{c}\right)_{+}\left(\sqrt{\theta_{\mathrm{eff}}}-\sqrt{\theta_{c}}\right) .
$$

This definition can be seen as a generalization of the Ashida-Michiue model for arbitrarily sediment sloping beds, see equation (1.6).

Other possibilities for the definition of $h_{m}$ can be found in the bibliography. For instance, it is possible to define (see [55])

$$
h_{m}=\frac{k_{1} d_{s}}{(1-\varphi)} \frac{\left(\theta_{\mathrm{eff}}-\theta_{c}\right)_{+}^{3 / 2}}{\sqrt{\theta_{\mathrm{eff}}}-k_{2} \sqrt{\theta_{c}}},
$$

$k_{1}$ and $k_{2}$ being two parameters of the model. Note that (2.32) is an approximation of (2.29), which is valid except near the threshold of motion. 


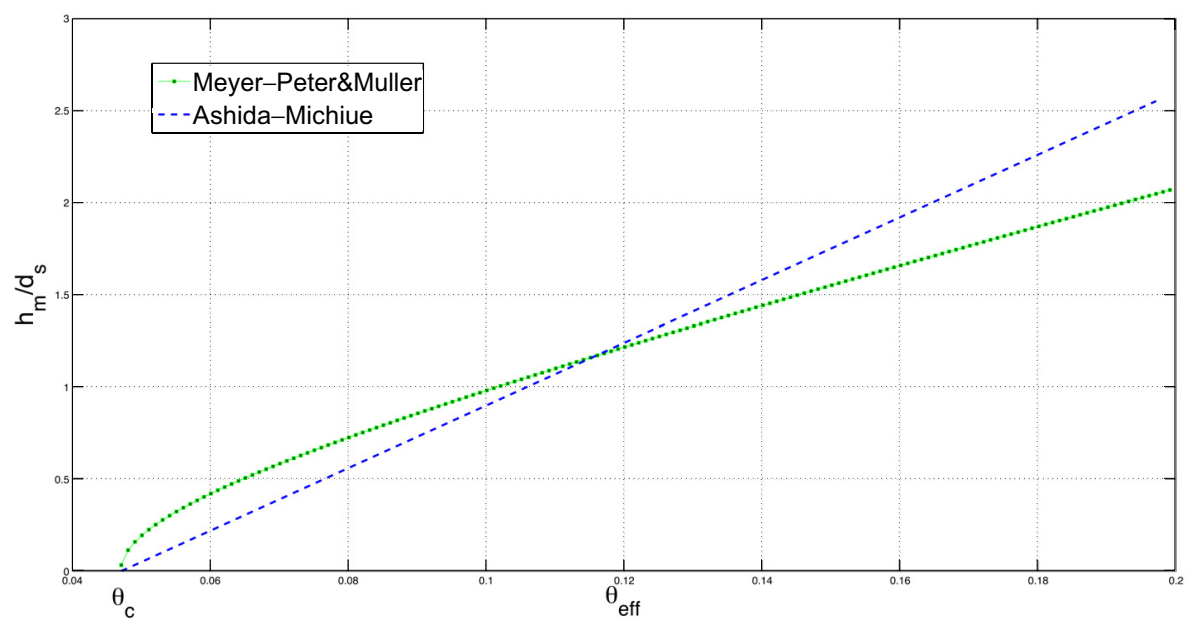

Figure 2. Definitions of $h_{m}$ corresponding to the Meyer-Peter and Müller and the Ashida-Michiue models given by equations (2.29) and (2.32).

Let us also remark that for the case $k_{2}=1$ and $v_{b}$ defined by (2.27) we obtain a SVE model with the following definition of the solid transport discharge,

$$
\frac{q_{b}}{Q}=\operatorname{sgn}\left(\tau_{\mathrm{eff}}^{(Q F)}\right) \frac{k_{1}}{(1-\varphi)}\left(\theta_{\mathrm{eff}}-\theta_{c}\right)_{+}^{3 / 2}
$$

This definition can be seen as a generalization of the Meyer-Peter and Müller model for arbitrarily sediment sloping beds, see equation (1.5).

Remark 2.3. The simplified model defined by (2.33) coincides with the classic Meyer-Peter and Müller model for $k_{1}=8$ and by neglecting all gravitational terms in the definition of $\tau_{\text {eff }}$. That is, for $\tau_{\text {eff }}$ defined by $(2.24)$ with $\Phi=\operatorname{sgn}\left(u_{1}\right)\left(\vartheta \frac{d_{s}}{h_{m}} \theta^{(Q F)}\right)^{1 / 2}$, where $\theta^{(Q F)}$ is defined by $(2.20)$.

We obtain also that with this definition of $\tau_{\text {eff }}$ the simplified model (2.31) coincides with the classic AshidaMichiue model when $K_{e} / K_{d}=17$.

By analogous arguments we can deduce a large range of classic models. This allows us to see classic models as simplified versions deduced from an asymptotic expansion of the Navier-Stokes equations, by considering the coupling between a shallow water layer and a Reynolds one. Thus, for example - see previous remark -, we can deduce that Meyer-Peter and Müller model corresponds to define $h_{m}$ by $(2.32)$ with $k_{1}=8$ and $k_{2}=1$. By while the Ashida-Michiue model corresponds to define $h_{m}$ by (2.29) with $K_{e} / K_{d}=17$. In Figure 2 we can see the comparison of both definitions of $h_{m}$ for $\theta_{c}=0.047$. Where we can observe that both definitions of $h_{m}$ are close.

\subsection{Energy balance of the models}

In this subsection we present the main result regarding the energy balance associated to the models $(L F)$ and $(Q F)$ presented before. In particular we prove that they admit an exactly dissipation energy. The proof of the theorem follows analogously as in [23], but for the sake of completeness, it is given in Appendix B.1. 
Theorem 2.4. The model (2.8) has a dissipative energy balance. More explicitly:

- for the $(L F)$ model:

$$
\begin{aligned}
& \partial_{t}\left(\frac{1}{2} r g\left(h_{1}+h_{2}+b\right)^{2}+\frac{1}{2} r h_{1}\left|u_{1}\right|^{2}\right)+\operatorname{div}_{x}\left(r h_{1} u_{1}\left(\frac{\left|u_{1}\right|^{2}}{2}+g\left(h_{1}+h_{2}+b\right)\right)\right) \\
& \quad+\operatorname{div}_{x}\left(h_{m} g\left(r h_{1}+h_{2}+b\right)\left(u_{1}-h_{m} \frac{\vartheta}{1-r} \mathcal{P} \sqrt{(1 / r-1) g d_{s}}\right)\right) \\
& \leq-g h_{m} \frac{\vartheta}{1-r} \sqrt{(1 / r-1) g d_{s}}|\mathcal{P}|^{2}-g h_{m}(1-r) \tan \delta \sqrt{(1 / r-1) g d_{s}}\left|v_{b}\right|,
\end{aligned}
$$

- for the $(Q F)$ model:

$$
\begin{aligned}
& \partial_{t}\left(\frac{1}{2} r g\left(h_{1}+h_{2}+b\right)^{2}+\frac{1}{2} r h_{1}\left|u_{1}\right|^{2}\right)+\operatorname{div}_{x}\left(r h_{1} u_{1}\left(\frac{\left|u_{1}\right|^{2}}{2}+g\left(h_{1}+h_{2}+b\right)\right)\right) \\
& \quad+\operatorname{div}_{x}\left(h_{m} g\left(r h_{1}+h_{2}+b\right)\left(u_{1}-h_{m} \sqrt{\frac{\vartheta}{1-r}}|\mathcal{P}|^{1 / 2} \operatorname{sgn}(\mathcal{P}) \sqrt{(1 / r-1) g d_{s}}\right)\right) \\
& \leq-g h_{m} \sqrt{\frac{\vartheta}{1-r}} \sqrt{(1 / r-1) g d_{s}}|\mathcal{P}|^{3 / 2}-g h_{m}(1-r) \tan \delta \sqrt{(1 / r-1) g d_{s}}\left|v_{b}\right|,
\end{aligned}
$$

where $\mathcal{P}$ is defined in $(2.9)$.

Remark 2.5. The right hand side of the energy balance (2.34) corresponds to

$$
-r C\left|u_{1}-u_{2}\right|^{2}-g(1-r) h_{m}\left|u_{2}\right| \tan \delta
$$

for the case of a linear friction law, where $u_{2}=v_{b}^{(L F)} \sqrt{(1 / r-1) g d_{s}}, v_{b}^{(L F)}$ is defined by (2.10) and $C$ by (2.4). For the quadractic friction law, the right hand side of $(2.35)$ corresponds to

$$
-r C_{1}\left|u_{1}-u_{2}\right|^{3}-g(1-r) h_{m}\left|u_{2}\right| \tan \delta
$$

where $u_{2}=v_{b}^{(Q F)} \sqrt{(1 / r-1) g d_{s}}, v_{b}^{(Q F)}$ is defined by $(2.11)$ and $C_{1}$ by $(2.6)$.

Note also that (2.36) and (2.37) correspond to the right hand side in the energy balance associated to the two-layer shallow water system, when we consider a friction law between layers (linear or quadratic, respectively) and a Coulomb friction law for the lower layer (see [22]).

\subsection{Energy balance of the classic Saint-Venant-Exner model}

As mentioned in the introduction there exist in the literature some simple solid transport formulae for which the corresponding SVE model has an associated dissipative energy equation. Nevertheless, up to our knowledge, no general result exists in the bibliography in this sense.

In this subsection we present a result that shows that a correction in the friction law in the momentum equation of the Saint-Venant system allows us to obtain a dissipative energy balance for any classic SVE model, provided that the solid transport discharge can be written under the general formulation (1.2). The proof of the Theorem can be found in Appendix B.2.

Theorem 2.6. Let us consider the general SVE system defined by

$$
\left\{\begin{array}{l}
\partial_{t} h_{1}+\operatorname{div}_{x} q_{1}=0, \\
\partial_{t} q_{1}+\operatorname{div}_{x}\left(\frac{\left|q_{1}\right|^{2}}{h_{1}}+\frac{1}{2} g h_{1}^{2}\right)+g h_{1} \nabla_{x}\left(h_{2}+b\right)+\hat{\tau} / \rho_{1}=0 \\
\partial_{t} h_{2}+\operatorname{div}_{x} q_{b}=0
\end{array}\right.
$$


$q_{b}$ being some solid transport discharge given by the general formulation (1.2) and with the following modification of the friction term:

$$
\frac{\hat{\tau}}{\rho_{1}}=\frac{\tau}{\rho_{1}}+\frac{1}{r} \xi_{m} \sqrt{g \zeta\left(h_{1}\right)} \mathcal{R}, \quad \text { with } \quad \mathcal{R}=g \nabla_{x}\left(r h_{1}+h_{2}+b\right),
$$

and $\tau$ defined by (1.3). Then, the SVE model satisfies the dissipative energy balance:

$$
\begin{aligned}
\partial_{t} & \left(\frac{1}{2} r h_{1}\left|u_{1}\right|^{2}+\frac{1}{2} r g\left(h_{1}+h_{2}+b\right)^{2}\right)+\operatorname{div}_{x}\left(r h_{1} u_{1}\left(\frac{\left|u_{1}\right|^{2}}{2}+g\left(h_{1}+h_{2}+b\right)\right)\right) \\
& +\operatorname{div}_{x}\left(\xi_{m} u_{1} \sqrt{g \zeta\left(h_{1}\right)} g\left(r h_{1}+h_{2}+b\right)\right) \leq-r g \zeta\left(h_{1}\right)\left|u_{1}\right|^{3},
\end{aligned}
$$

where

$$
\frac{\xi_{m}}{d_{s}}=\frac{1}{1-\varphi} k_{1} \theta^{m_{1}-1 / 2}\left(\theta-k_{2} \theta_{c}\right)_{+}^{m_{2}}\left(\sqrt{\theta}-k_{3} \sqrt{\theta_{c}}\right)_{+}^{m_{3}} .
$$

Remark 2.7. Note that the previous result also implies that the classic SVE model, that is, if we set $\hat{\tau}=\tau$, verifies a dissipative energy inequality up to the order of $\mathcal{O}(\hat{\tau}-\tau)=\mathcal{O}\left(\xi_{m}\right)$. And $\xi_{m}$ approximates the thickness of the moving sediment layer (see [24]), which is in many situations a very small quantity.

\section{Numerical tests}

In this section we present three numerical tests, for the simplified model obtained from (2.8) with (2.32), that is, for the solid transport equation given by (2.33). This coincides with a classic Meyer-Peter and Müller model with a modified shear stress given by $\tau_{\text {eff }}$.

In the first test we study the evolution of a dune for different values of the the repose angle. The purpose of the second test is to compare the influence of the deduced modification in the definition of the effective shear stress respect to classic ones. A comparison with experimental data is presented in the third test.

The numerical results follow from a combination of the scheme described in [6] with a discrete approximation of bottom and surface derivatives. The numerical simulations are done with a CFL number equal to 0.5.

\subsection{Test 1}

In this first test we propose to study the influence of $\tan \delta=\theta_{c} / \vartheta$ in (2.15). To do so, let us consider the following initial condition

$$
h_{2}(0, x)=\left\{\begin{array}{ll}
0.2, & \text { if } x \in[4,6], \\
0.1, & \text { otherwise }
\end{array} \quad h_{1}(0, x)+h_{2}(0, x)=1, \quad q_{1}(0, x)=1.5 .\right.
$$

The initial condition is shown in Figure 3a. We set the left boundary condition $q(t, 0)=1.5$, and open boundary conditions on the right hand side.

The parameters for the model have been set as follows

$$
\begin{gathered}
r=0.34, \quad d_{s}=10^{-3}, \\
\varphi=0, \quad K_{e}=10, \quad K_{d}=1, \quad \theta_{c}=0.047
\end{gathered}
$$

with the Manning friction law and as Manning coefficient $n=0.01$. The computational domain used is $[0,10]$ with 800 points.

Remark that this is a rather severe test: the bottom is discontinuous and thus we have $\partial_{x}\left(h_{2}+b\right)= \pm \infty$ initially. We run the test for different values of $\delta$ ranging from $25^{\circ}$ to $89^{\circ}$. In Figures $3 \mathrm{~b}$ and $3 \mathrm{c}$ we show the free surface and the sediment bottom surface at time $t=2000$ for $\delta=89^{\circ}, 60^{\circ}, 45^{\circ}$ and $25^{\circ}$. The results are 


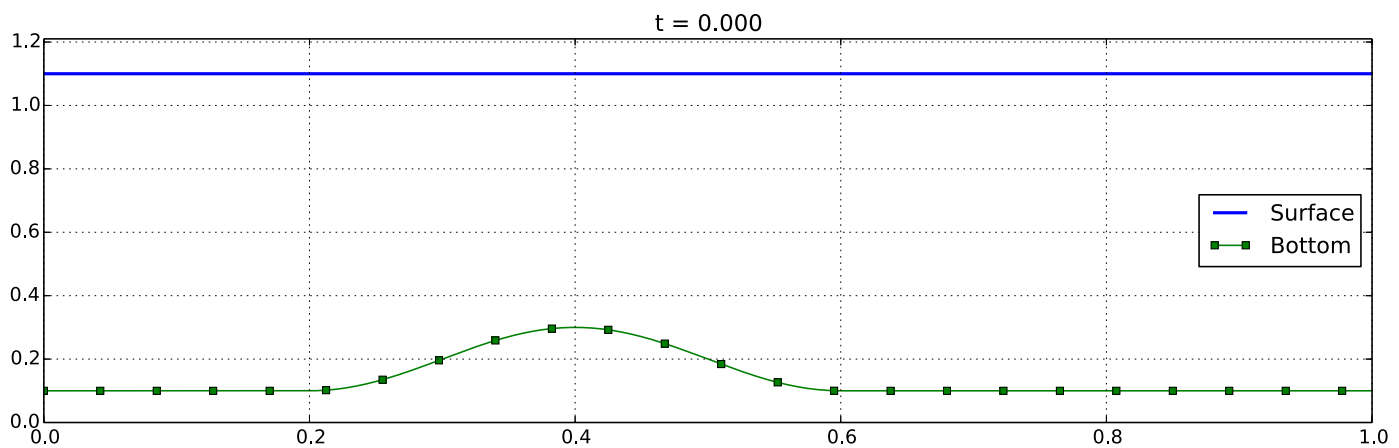

(a) Test 1: Initial condition

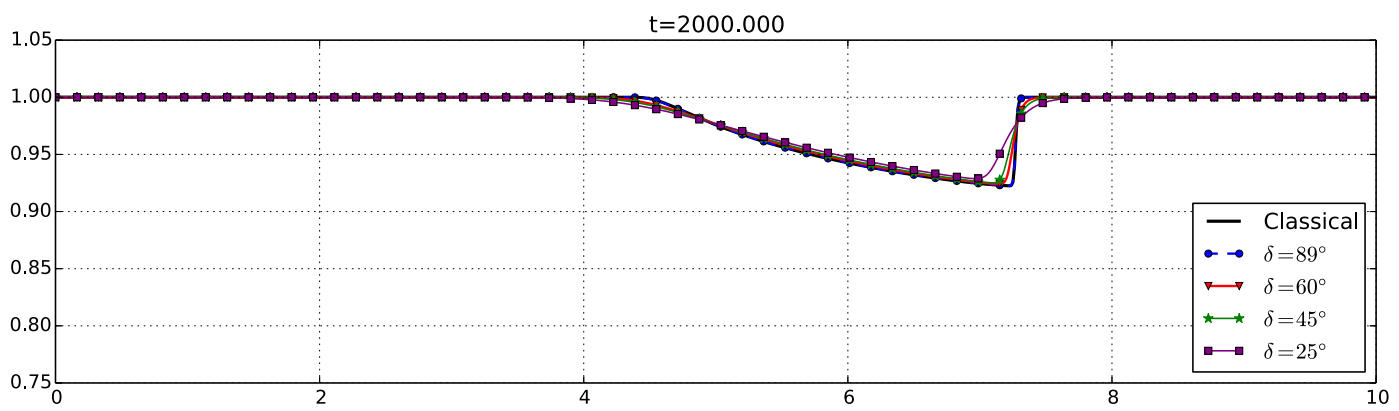

(b) Test 1: Surface at time $t=2000$

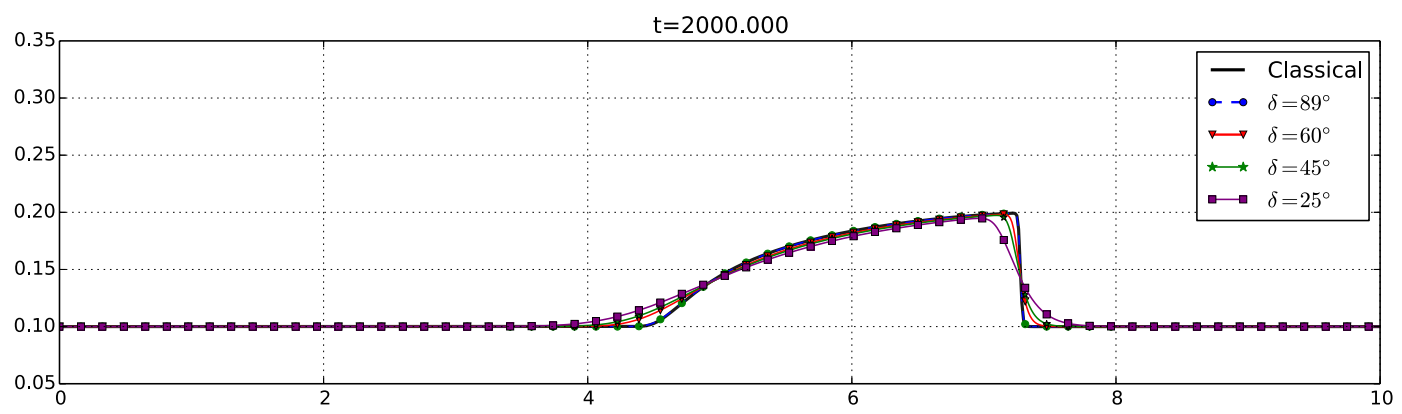

(c) Test 1: Bottom at time $t=2000$

Figure 3. Test 1: Initial condition and evolution for different values of $\delta$.

also compared with a classic Meyer-Peter and Müller model. A more detailed comparison of the final bottom can be seen in Figure 4a.

Remark that for $\delta \rightarrow 90^{\circ}$ we have $\vartheta \rightarrow 0$. Thus, the model reduces to a classic Meyer-Peter and Müller model for values of $\delta$ near $90^{\circ}$ as it can be observed in Figures 3c and 4a. For smaller values of $\delta$, the effects of the gradient $\partial_{x}\left(r h_{1}+\eta\right)$ play a relevant role in the stress tensor. This is shown, for instance, in Figures $4 \mathrm{~b}$ and $4 \mathrm{c}$, where the value $\tau-\tau_{\text {eff }}$ is displayed. The steep profile of $\partial_{x}\left(r h_{1}+\eta\right)$ makes this term play an essential role in the evolution of the test. The smaller the value $\delta$, the bigger the influence of the gradient on this test. This is specially true for small values of $t$. This results in a smoothing of the dune profile, and in particular in the advancing front of the dune. 


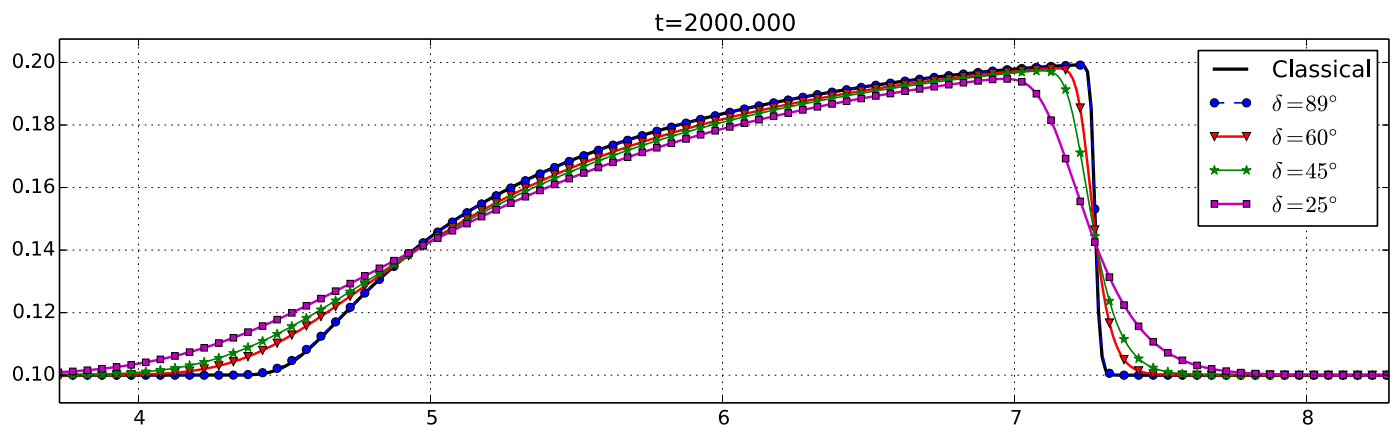

(a) Test 1: Bottom comparison (Zoom)

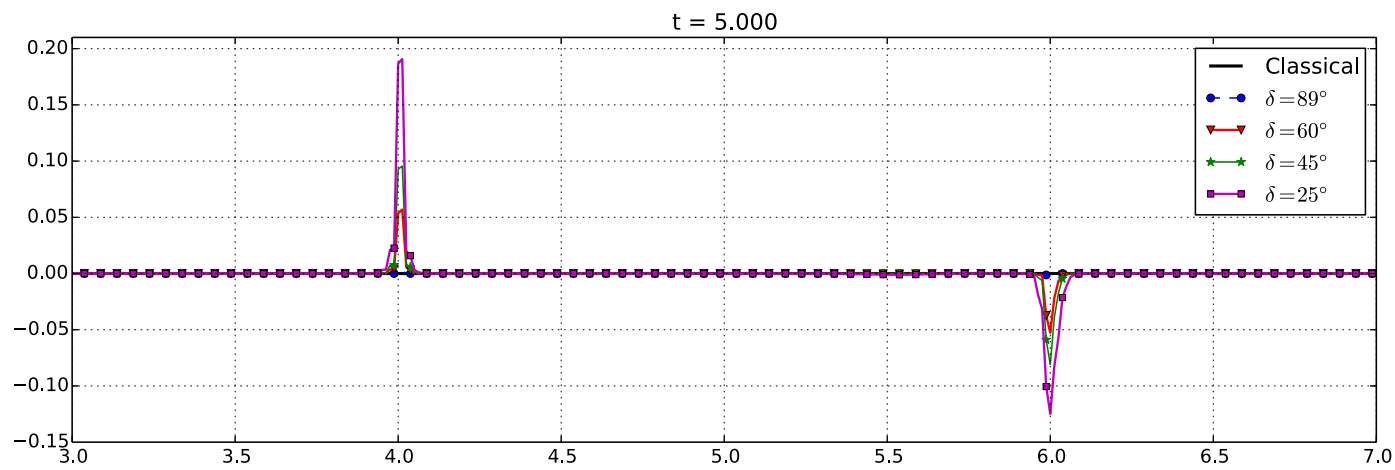

(b) Test 1: $\tau-\tau_{\text {eff }}$ at time $t=5$ (Zoom)

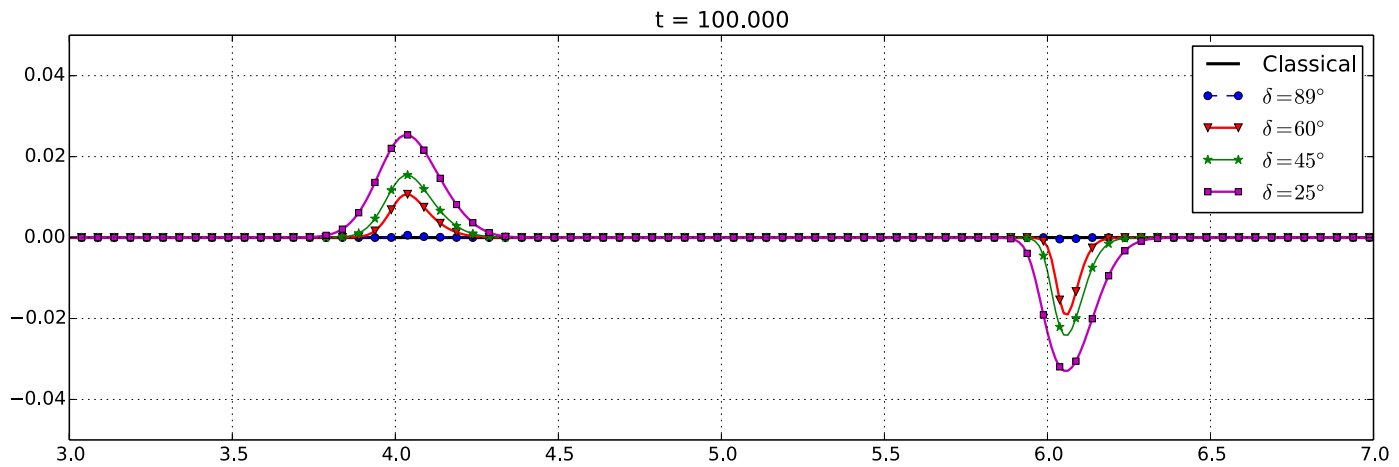

(c) Test 1: $\tau-\tau_{\text {eff }}$ at time $t=100$ (Zoom)

Figure 4. Test 1: Influence of $\delta$.

\subsection{Test 2}

The purpose of this test is to study the influence on the definition of the effective shear stress in terms of $\nabla_{x} \eta$ instead of $\nabla_{x}\left(r h_{1}+\eta\right) /(1-r)$. Following Remark 2.1, both terms can be related by

$$
\nabla_{x} \eta=\frac{1}{1-r} \nabla_{x}\left(r h_{1}+\eta\right)-\frac{r}{1-r} \nabla_{x}\left(b+h_{1}+h_{2}\right) .
$$

This means that both definitions coincide only for constant free surface. We recall that the model proposed in [26] defines the effective shear in terms of $\nabla_{x} \eta$. This model was also studied in [39]. 


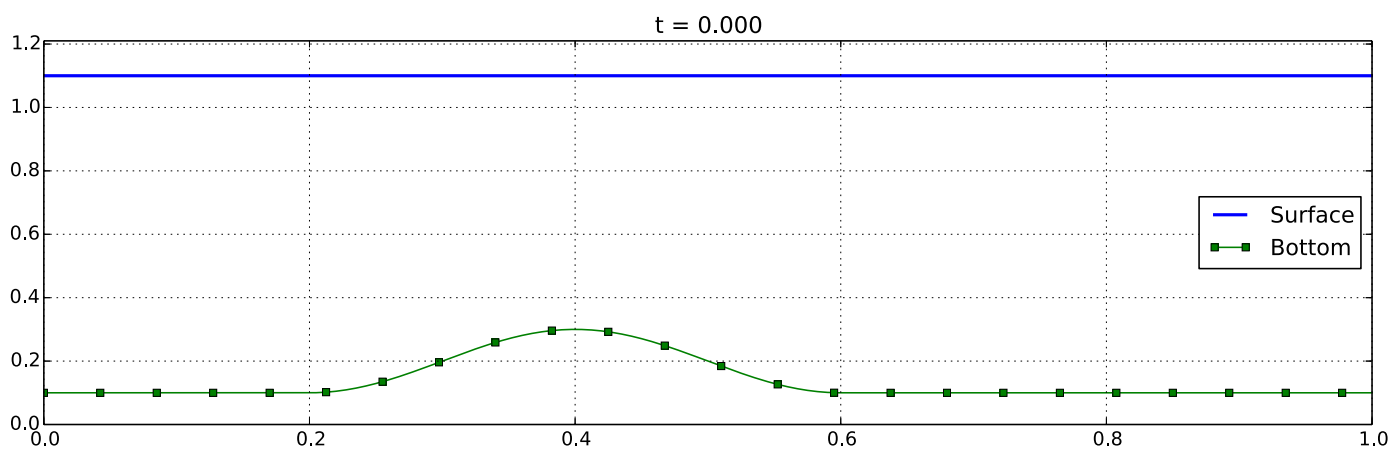

Figure 5. Test 2: Initial condition.

We set as initial condition

$$
\begin{aligned}
& h_{2}(0, x)= \begin{cases}0.1+0.1\left(1+\cos \left(\frac{x-0.4}{0.2 \pi}\right)\right), & \text { if } x \in[0.2,0.6], \\
0.1, & \text { otherwise }\end{cases} \\
& h_{1}(0, x)+h_{2}(0, x)=1, \quad q_{1}(0, x)=1.4 .
\end{aligned}
$$

The initial condition is shown in Figure 5.

We set the boundary condition $q(t, 0)=1.4$ and we have used the same parameters and computational domain described in Test $1 . \delta$ is fixed to $45^{\circ}$.

Figures $6 \mathrm{a}$ and $6 \mathrm{~b}$ show the difference observed on the surface and bottom when the different terms $\partial_{x}\left(r h_{1}+\eta\right)$ and $\partial_{x} \eta$ are used in the definition of $\tau_{\text {eff }}$. Due to the shape of the surface and bottom, we get that $\partial_{x} \eta \geq$ $\frac{1}{1-r}\left(r h_{1}+\eta\right)$ and $\partial_{x} \eta \leq \frac{1}{1-r}\left(r h_{1}+\eta\right)$ on the upstream and downstream part of the dune respectively which is shown in 6c. This makes the influence of the gravitational effects in $\tau_{\text {eff }}$ stronger when $\partial_{x} \eta$ is used and results as a more diffused shape observed in Figure 6b.

\subsection{Test 3}

In this section we present a comparison with experimental data obtained by the Hydraulic Laboratory of Escuela Superior de Ingenieros de Caminos, Canales y Puertos (A Coruña University) over a channel of $15 \mathrm{~m}$ long and $0.5 \mathrm{~m}$ width (for more details see $[6,48]$ ). We compare the experimental data with the numerical simulation corresponding to the modified Meyer-Peter and Müler's model, defined by (2.33).

The experimental test was developed by introducing a sand layer in the central part of laboratory channel, and inducing hydrodynamical conditions to erode the sand layer. The channel has slope of $0.052 \%$. Sand layer was situated in interval $[4.5 \mathrm{~m}, 9 \mathrm{~m}]$, with a thickness of $4.5 \mathrm{~cm}$; being the average diameter of the grain equals to $1 \mathrm{~mm}$. As boundary conditions, an incoming discharge equal to $0.0285 \mathrm{~m}^{2} / \mathrm{s}$ upstream is imposed. The water thickness is $0.129 \mathrm{~m}$ downstream.

We have the experimental measurements of the sediment profile at several points for $t=10, t=40$ and $t=120 \mathrm{~min}$. For the numerical simulation we have considered as initial condition for the sediment surface a profile obtained by an interpolation of the data at $t=10 \mathrm{~min}$ (see Fig. 7). The initial condition for the free surface and the discharge can be precomputed by considering this profile of the sediment layer and previous boundary conditions.

For numerical simulation we have meshed the domain with 250 nodes. The CFL is set to 0.9. Sediment porosity is set to 0.4. Friction between fluid and bed is modeled using a Manning's law with coefficient equal to 0.0125 over the fixed bed and 0.0196 over the sediment layer. 


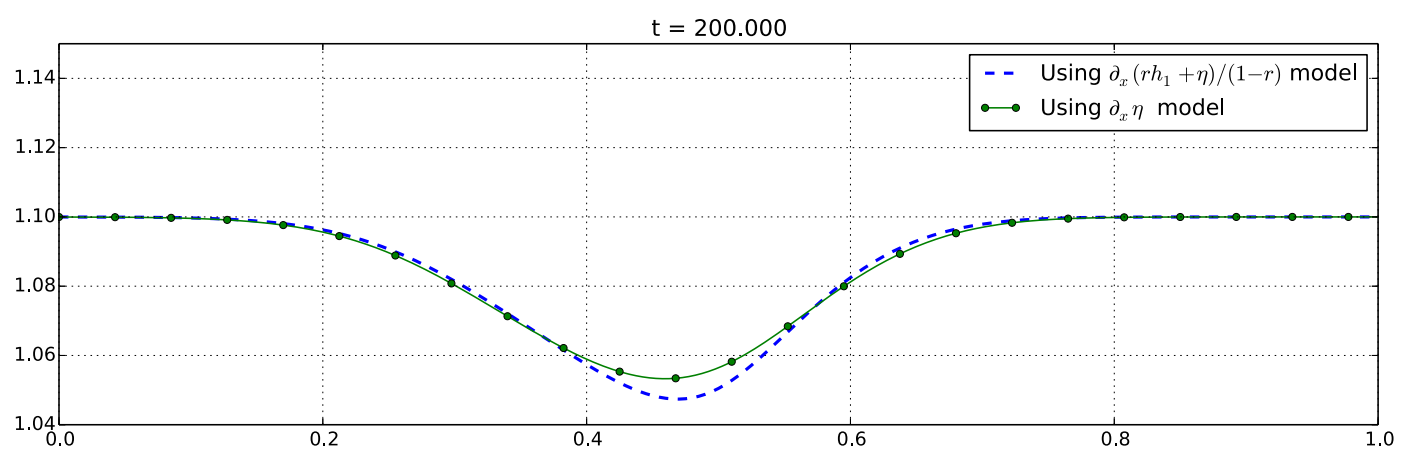

(a) Test 2: Surface at time $t=200$

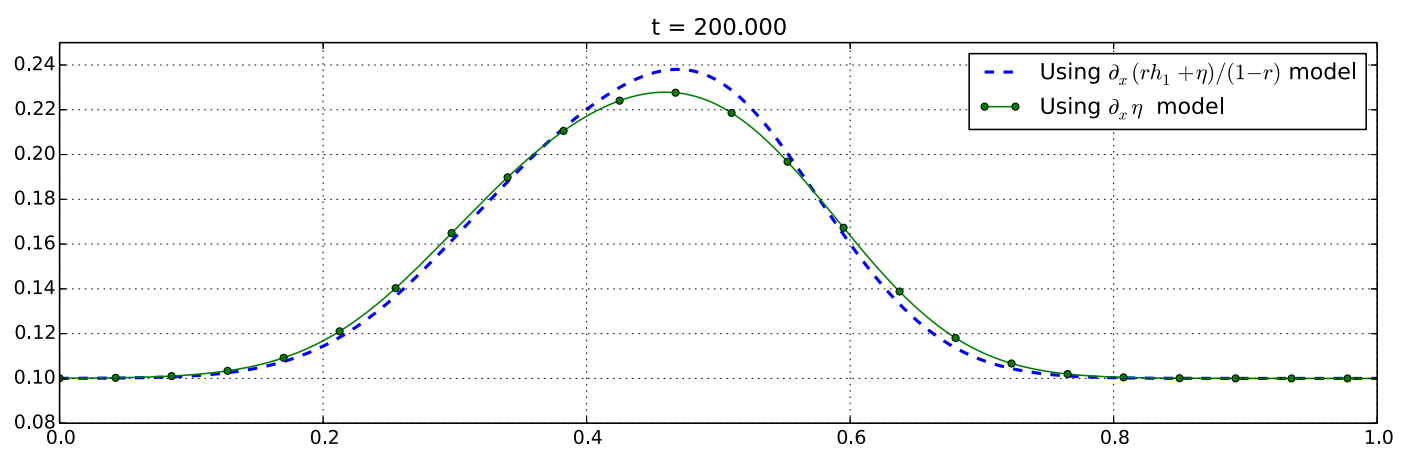

(b) Test 2: Bottom at time $t=200$

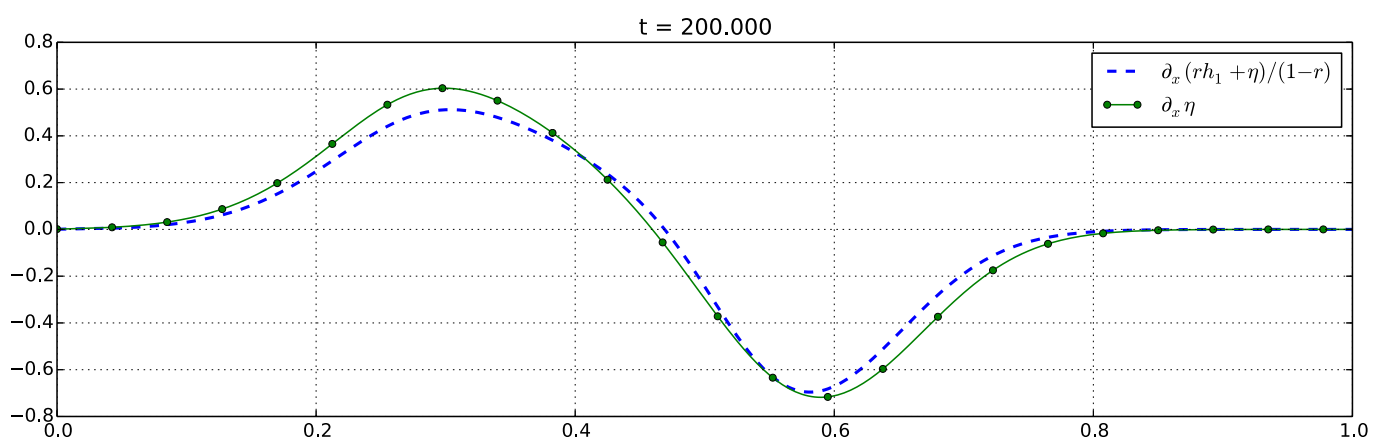

(c) Test 2: $\partial_{x}\left(r h_{1}+\eta\right)$ and $\partial_{x} \eta$ at time $t=200$

Figure 6. Test 2: Comparisons at time $t=200$.

Comparisons with the experimental data for $t=40$ and $t=120$ minutes are presented in Figure 8. Three numerical simulations have been done, corresponding to the Coulomb friction angle $\delta=10^{\circ}, \delta=22^{\circ}$ and $\delta=89^{\circ}$. We can observe that in both cases the averaged position of the sediment surface is well reproduced by the numerical simulations. We can remark that at $t=120$ minutes the numerical simulation reproduces correctly the position of the discontinuity in the profile of the sediment bed, located at $x \approx 10.7$. Nevertheless, the model does not produce the small sediment bed at the right of the shock. Although, we have tested a large range of models to define the solid transport discharge and any of them reproduce this advance of the sediment bed at the right of the shock. Which is probably a purely tridimensional effect. 


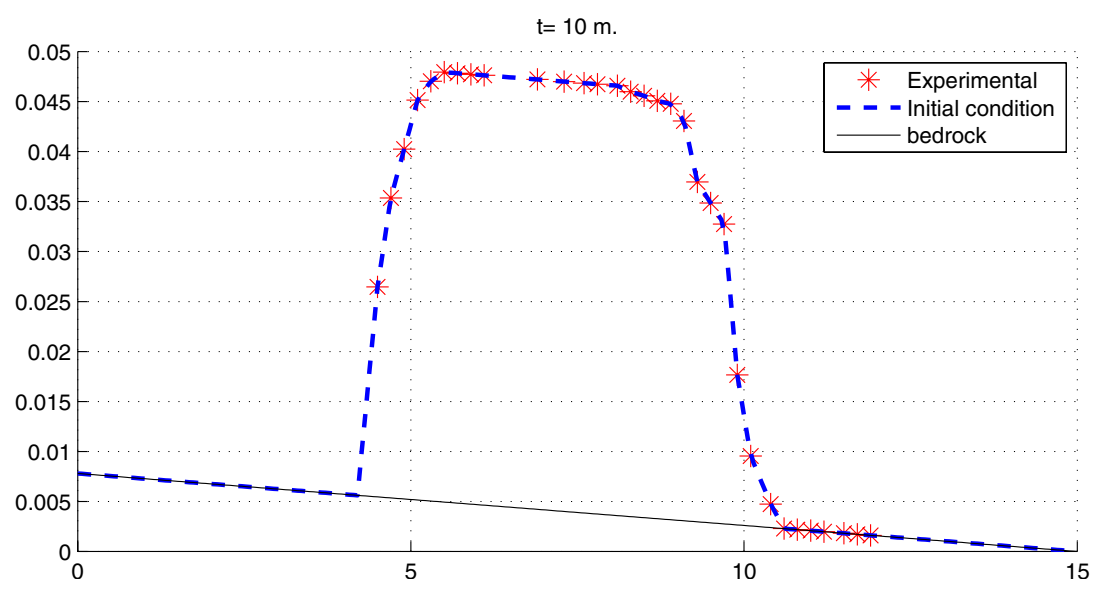

Figure 7. Test 3: Sediment surface. Initial condition.
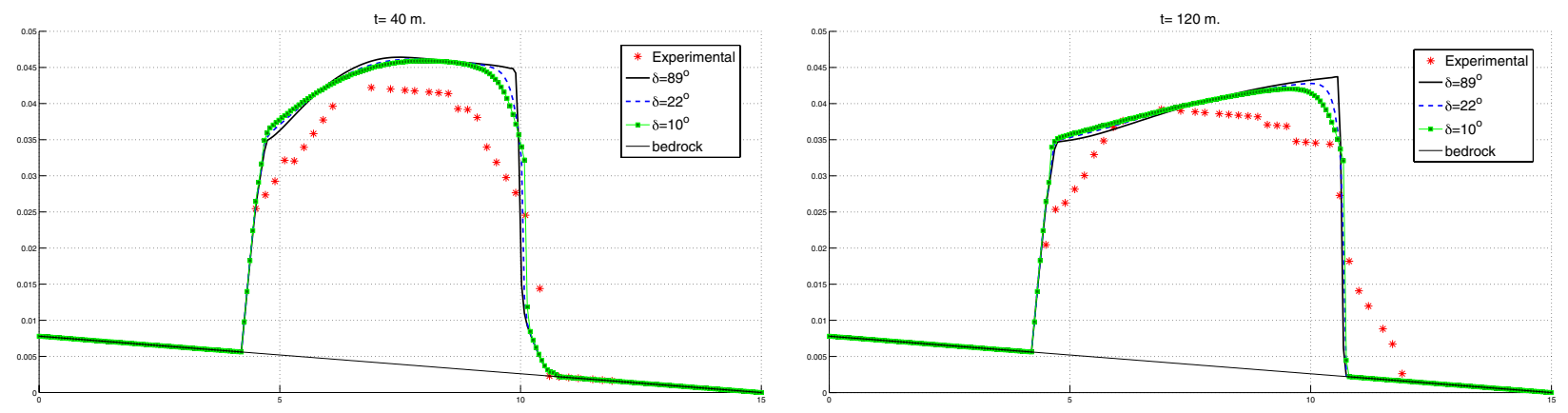

Figure 8. Test 3: Sediment surface evolution at $t=40 \mathrm{~min}$. and $t=120 \mathrm{~min}$.

\section{Conclusions}

The SVE model has been deduced in this work by an asymptotic analysis of the Navier-Stokes equations. We show that depending on the considered friction law at the interface between the fluid and the sediment we can obtain different definitions of solid transport discharge. Results have been presented for the case of linear and quadratic friction laws. The stop criterion, which is usually represented as a positive part in terms of a critical Shields stress, is deduced by including a Coulomb friction law in the internal interface of the sediment layer.

The proposed models have some advantages with respect to classic ones: they have an associated dissipative energy, they are deduced for arbitrarily sediment sloping beds and they depend on the thickness of the moving sediment layer, which implies sediment mass conservation in general situations.

Moreover, from the deduction of the model we obtain a modification on the definition of the effective shear stress. We obtain that it should be defined in terms of the gradient of total pressure, $\nabla_{x}\left(r h_{1}+b+h_{2}\right)$ instead of the gradient of sediment bed, $\nabla_{x}\left(b+h_{2}\right)$, as it is usually done. Nevertheless, by considering this classic definitions we cannot obtain a model with a dissipative energy.

The proposed model depends on the definition of the sediment transference term $T_{m}$. This term represents the difference between erosion and deposition rates at the internal interface of the sediment layer, which separates static from moving particles. For uniform flows, where $T_{m}=0$, we obtain a generalization of classic solid transport discharge formulae by including gravitational effects. Nevertheless, let us remark that the definition of $T_{m}$ is of great importance in the closure of the model and to suppose $T_{m}=0$ (that is, to use classic formulae) 
constitutes a significant simplification in the definition of the solid transport discharge, and consequently in its range of application (see [24]).

Finally, the deduction of the model and the proof of the existing energy dissipation equation provides important information on classic models. It allows us to understand why classic SVE models do not have an associated energy. Moreover, in order to have an associated dissipative energy we may add an extra term - a term that is deduced in the modelling process - in the definition of the shear stress in the momentum equation of the Saint-Venant model.

In the numerical tests we presented the evolution of a dune for several repose angle. We can see that the expected morphodynamical form of the shape of dune is well reproduced. Moreover, the vertical profile of the advancing front obtained with classic models is avoided. The influence of the definition of the effective shear stress proposed here with respect to the classic one is compared in the second test. We can observe that a difference appears between both definitions just in the area over the dune, where the free surface is not constant. In the last numerical test we have compared the generalization of the Meyer-Peter and Müller model with experimental data. We obtain that the averaged position of the dune is well captured in comparison with experimental data. The position of the shock is also well approximated.

\section{Appendix A: Derivation of THE SEDIMENT TRANSPORT MODELS}

In this section we develop the derivation of the sediment transport models from an asymptotic analysis of the Navier-Stokes equations. These processes are different for each layer. In [23] a similar development is performed coupling also shallow water and Reynolds equations in a multiscale framework but for a transport of pollutant problem. Thus, we consider this work as a reference in the development, which in turn is based on the original works $[30,43]$.

The derivation is divided in several steps: first, we write the initial system in non-dimensional form; next we make an hydrostatic approximation, we assume a suitable asymptotic regime and we average the equations out.

\section{Dimensionless equations}

To begin with, we write the equations and boundary conditions under dimensionless form. We set the dimensionless variables, where we must take into account the different nature of the fluids in two layers, so we make it separately. Indeed the main property that we want to point out is the different order of the velocities, as we have discussed in Section 2.1. Since we study a coupled system we relate the two characteristic velocities by the aspect ratio to indicate that the sediment layer is slower than the fluid layer.

We denote by $H$ and $L$ the characteristic height and length respectively. To impose the shallow flow condition, we assume that the aspect ratio between the characteristic height and length is small, as usual we denote it by $\varepsilon=\frac{H}{L}$. The characteristic kinematic viscosity for layer 2 is denoted by $M_{2}$, since this viscosity is not a constant. The characteristic velocities are $U$ for the layer 1 and $U_{2}$ for the sediment layer, consequently, the characteristic times are respectively $T=\frac{L}{U}$ and $T_{2}=\frac{L}{U_{2}}$ for each layer. In particular we assume that

$$
U_{2}=\varepsilon^{2} U, \quad \text { so consequently } \quad T_{2}=\frac{L}{\varepsilon^{2} U}=\frac{1}{\varepsilon^{2}} T .
$$

This hypothesis also affects the definitions of the Froude and Reynolds numbers. For the sake of clarity we indicate separately these variables. We consider the "star" notation for the dimensionless variables.

General dimensionless variables:

$$
\begin{array}{ll}
x=L x^{*} & z=H z^{*} \quad h_{i}=H h_{i}^{*} b=H b^{*} \\
\text { fric }_{f}=\rho_{2} U^{2} \text { fric }_{f}^{*} & \text { fric }=\rho_{1} U^{2} \text { fric }^{*}
\end{array}
$$


Nondimensionalization for layer 1:

$$
\begin{array}{lll}
u_{1}=U u_{1}^{*} & w_{1}=\varepsilon U w_{1}^{*} \quad t=\frac{L}{U} t_{1}^{*} \quad p_{1}=\rho_{1} U^{2} p_{1}^{*} \\
R e_{1}=\frac{U L}{\nu_{1}} & F r_{1}=\frac{U}{\sqrt{g H}} \quad \mu_{1}=\rho_{1} \nu_{1}
\end{array}
$$

Nondimensionalization for layer 2:

$$
\begin{array}{llll}
u_{2}=\varepsilon^{2} U u_{2}^{*} & w_{2}=\varepsilon^{3} U w_{2}^{*} & t=\frac{L}{\varepsilon^{2} U} t_{2}^{*} & p_{2}=\frac{\varepsilon \rho_{2} M_{2} U}{H} p_{2}^{*} \\
R e_{2}=\varepsilon^{2} \frac{U L}{M_{2}} & F r_{2}=\varepsilon^{2} \frac{U}{\sqrt{g H}} & \mu_{2}=\rho_{2} M_{2} \mu_{2}^{*} & T_{m}=\varepsilon^{3} U T_{m}^{*}
\end{array}
$$

We also define the ratio of densities,

$$
r=\frac{\rho_{1}}{\rho_{2}}<1
$$

Thus, the equations and the boundary conditions written in dimensionless form read as follows (we omit the "star" to simplify the notation):

$$
\begin{gathered}
\left\{\begin{array}{l}
\partial_{t_{1}} u_{1}+\operatorname{div}_{x}\left(u_{1} \otimes u_{1}\right)+\partial_{z}\left(u_{1} w_{1}\right)-\frac{2}{R e_{1}} \operatorname{div}_{x}\left(D_{x} u_{1}\right) \\
-\frac{1}{\varepsilon^{2}} \frac{1}{R e_{1}} \partial_{z}^{2} u_{1}-\frac{1}{R e_{1}} \nabla_{x}\left(\partial_{z} w_{1}\right)+\nabla_{x} p_{1}=0 ; \\
\partial_{t_{1}} w_{1}+u_{1} \nabla_{x} w_{1}+w_{1} \partial_{z} w_{1}-\frac{1}{R e_{1}} \operatorname{div}_{x}\left(\nabla_{x} w_{1}\right) \\
-\frac{1}{\varepsilon^{2}} \frac{1}{R e_{1}} \partial_{z}\left(\operatorname{div}_{x} u_{1}\right)-2 \frac{1}{\varepsilon^{2}} \frac{1}{R e_{1}} \partial_{z}^{2} w_{1}+\frac{1}{\varepsilon^{2}} \partial_{z} p_{1}=-\frac{1}{\varepsilon^{2}} \frac{1}{F r_{1}^{2}} ; \\
\operatorname{div}_{x} u_{1}+\partial_{z} w_{1}=0 .
\end{array}\right. \\
\left\{\begin{array}{l}
\varepsilon^{2} \operatorname{Re}_{2}\left(\partial_{t_{2}} u_{2}+\operatorname{div}_{x}\left(u_{2} \otimes u_{2}\right)+\partial_{z}\left(u_{2} w_{2}\right)\right) \\
=-\nabla_{x} p_{2}+\partial_{z}\left(\mu_{2} \partial_{z} u_{2}\right)+2 \varepsilon^{2} \operatorname{div}_{x}\left(\mu_{2} D_{x} u_{2}\right)+\varepsilon^{2} \nabla_{x}\left(\mu_{2} \partial_{z} w_{2}\right) ; \\
\varepsilon^{4} R_{2}\left(\partial_{t_{2}} w_{2}+u_{2} \nabla_{x} w_{2}+w_{2} \partial_{z} w_{2}\right) \\
=-\partial_{z} p_{2}+\varepsilon^{2}\left(2 \partial_{z}\left(\mu_{2} \partial_{z} w_{2}\right)+\partial_{z}\left(\mu_{2} \operatorname{div}_{x} u_{2}\right)+\varepsilon^{2} \operatorname{div}_{x}\left(\mu_{2} \nabla_{x} w_{2}\right)\right)-\varepsilon^{2} \frac{R e_{2}}{F r_{2}^{2}}
\end{array}\right. \\
\operatorname{div}_{x} u_{2}+\partial_{z} w_{2}=0
\end{gathered}
$$

- Conditions at the free surface:

$$
\begin{aligned}
\left(-\frac{2}{R e_{1}} D_{x} u_{1}+p_{1}\right) \nabla_{x} \eta_{s}+\frac{1}{R e_{1}} \nabla_{x} w_{1}+\frac{1}{\varepsilon^{2}} \frac{1}{R e_{1}} \partial_{z} u_{1}=0 \\
-\frac{1}{R e_{1}}\left(\varepsilon^{2} \nabla_{x} w_{1}+\partial_{z} u_{1}\right) \nabla_{x} \eta_{s}+\frac{2}{R e_{1}} \partial_{z} w_{1}-p_{1}=0
\end{aligned}
$$

- Conditions at the interface:

$$
\begin{aligned}
& \partial_{t_{1}} \eta+u_{1} \cdot \nabla_{x} \eta=w_{1} \\
& \partial_{t_{2}} \eta+u_{2} \cdot \nabla_{x} \eta=w_{2}
\end{aligned}
$$




$$
\begin{gathered}
r \varepsilon^{2}\left|\nabla_{x} \eta\right|^{2}\left(2 \frac{1}{R e_{1}} D_{x} u_{1}-p_{1}\right)+r \frac{1}{R e_{1}}\left(-2\left(\varepsilon^{2} \nabla_{x} w_{1}+\partial_{z} u_{1}\right) \nabla_{x} \eta+2 \partial_{z} w_{1}\right)-r p_{1} \\
=-\frac{1}{R e_{2}}\left(\varepsilon^{6}\left|\nabla_{x} \eta\right|^{2}\left(2 \mu_{2} D_{x} u_{2}-p_{2}\right)-2 \varepsilon^{4} \mu_{2}\left(\varepsilon^{2} \nabla_{x} w_{2}+\partial_{z} u_{2}\right) \nabla_{x} \eta+2 \varepsilon^{4} \mu_{2} \partial_{z} w_{2}+\varepsilon^{2} p_{2}\right) \\
\frac{1}{R e_{1}}\left(-2 \nabla_{x} \eta\left(D_{x} u_{1}-\partial_{z} w_{1}\right)+\left(\nabla_{x} w_{1}+\frac{1}{\varepsilon^{2}} \partial_{z} u_{1}\right)\left(1-\varepsilon^{2}\left|\nabla_{x} \eta\right|^{2}\right)\right)=\frac{1}{\varepsilon} \operatorname{fric} \sqrt{1+\varepsilon^{2}\left|\nabla_{x} \eta\right|^{2}} \\
\varepsilon^{4} \mu_{2} \frac{1}{R e_{2}}\left(-2 \nabla_{x} \eta\left(D_{x} u_{2}-\partial_{z} w_{2}\right)+\left(\nabla_{x} w_{2}+\frac{1}{\varepsilon^{2}} \partial_{z} u_{2}\right)\left(1-\varepsilon^{2}\left|\nabla_{x} \eta\right|^{2}\right)\right)=r \frac{1}{\varepsilon} \text { fric } \sqrt{1+\varepsilon^{2}\left|\nabla_{x} \eta\right|^{2}}
\end{gathered}
$$

- Conditions at the internal sediment interface:

$$
\begin{gathered}
\partial_{t_{2}} h_{f}+u_{2} \nabla_{x} \eta_{f}-w_{2}=-T_{m} \\
\varepsilon^{4} \mu_{2} \frac{1}{R e_{2}}\left(-2 \nabla_{x} \eta_{f}\left(D_{x} u_{2}-\partial_{z} w_{2}\right)+\left(\nabla_{x} w_{2}+\frac{1}{\varepsilon^{2}} \partial_{z} u_{2}\right)\left(1-\varepsilon^{2}\left|\nabla_{x} \eta_{f}\right|^{2}\right)\right)=\frac{1}{\varepsilon} \text { fric }_{f} \sqrt{1+\varepsilon^{2}\left|\nabla_{x} \eta_{f}\right|^{2}}
\end{gathered}
$$

- Condition at the bottom:

$$
-u_{2} \nabla_{x} b+w_{2}=0
$$

\section{Layer $\Omega_{1}$ : Shallow water}

In this section we obtain the mass and momentum approximated equations for the fluid layer. This derivation is well known, so we just give the hypothesis considered and the final result.

To develop the asymptotic analysis, we assume the following hypotheses on the data:

$$
\frac{1}{R e_{1}}=\varepsilon \nu_{01}, \quad \text { fric }=\varepsilon \text { fric }_{0} .
$$

Then we integrate this system in $\left[\eta, \eta_{s}\right]$ and use conditions (A.3), (A.4), (A.5), (A.6) and (A.9). The pressure reads,

$$
p_{1}(z)=-\frac{1}{F r_{1}^{2}}\left(z-\eta_{s}^{0}\right)-2 \varepsilon \nu_{01} \operatorname{div}_{x} u_{1}^{0} .
$$

The mass and momentum equations at first order (unknowns are then denoted with superscript 0) are:

$$
\begin{gathered}
\partial_{t} h_{1}^{0}+\operatorname{div}_{x}\left(h_{1}^{0} u_{1}^{0}\right)=0 . \\
\partial_{t_{1}}\left(h_{1}^{0} u_{1}^{0}\right)+\operatorname{div}_{x}\left(h_{1}^{0}\left(u_{1}^{0} \otimes u_{1}^{0}\right)\right)+\frac{1}{2} \frac{1}{F r_{1}^{2}} \nabla_{x}\left(h_{1}^{0}\right)^{2}+\frac{1}{F r_{1}^{2}} h_{1}^{0} \nabla_{x} \eta^{0}+\text { fric }_{0}=0,
\end{gathered}
$$

where the friction term fric $_{0}$ is specified later in relations (A.26) and (A.27) that gives:

$$
\text { fric }_{0}=\frac{1}{r} \frac{1}{F r_{1}^{2}} h_{m}^{0}\left((1-r) \operatorname{sgn}\left(u_{2}\right) \tan \delta_{0}+\left(r \nabla_{x} h_{1}^{0}+\nabla_{x} \eta^{0}\right)\right)
$$




\section{Layer $\Omega_{2}$ : Reynolds}

We derive in this section the first order approximation of the evolution equation for the layer 2. Due to the more complex dimensionless considered for this layer, it is necessary to keep in the derivation process the terms of order zero and one. Later we will only consider the terms of principal order. Like before, the unknowns at first order approximation are denoted with superscript 0 . The unknowns at second order approximations are denoted with "tilda".

We consider the following asymptotic regime in order to keep the gravitational and intergranular friction effects:

$$
\frac{\varepsilon^{2}}{R e_{2}}=\varepsilon^{-1} \nu_{02} ; \quad \operatorname{fric}_{f}=\varepsilon \operatorname{fric}_{f_{0}}
$$

(Remember that $R e_{2}=\varepsilon^{2} \frac{U L}{M_{2}}$.)

For simplicity we introduce the notation

$$
\beta_{0}=\varepsilon \frac{R e_{2}}{F r_{2}^{2}}=\frac{1}{\nu_{02} F r_{1}^{2}} .
$$

We write the momentum equations in (A.2) up to second order:

$$
\begin{aligned}
-\partial_{z}\left(\mu_{2} \partial_{z} u_{2}\right)+\nabla_{x} p_{2} & =\mathcal{O}\left(\varepsilon^{2}\right) \\
\partial_{z} p_{2}+\varepsilon \beta_{0} & =\mathcal{O}\left(\varepsilon^{2}\right) .
\end{aligned}
$$

Note that for the static layer we have that $u_{2}(z)=0$ for $b \leq z<\eta_{f}$. The mass equation for the sediment layer comes from the integration of the incompressibility equation using conditions (A.11) and (A.13) to get

$$
\partial_{t_{2}} h_{f}=-T_{m} .
$$

Using conditions (A.7) and (A.11) we write the mass conservation for the moving layer:

$$
\partial_{t_{2}} h_{m}+\operatorname{div}_{x}\left(\int_{\eta_{f}}^{\eta} u_{2} \mathrm{~d} z\right)=T_{m} .
$$

To obtain the pressure we integrate equation (A.19) for $z \in\left[\eta_{f}, \eta\right]$ :

$$
\tilde{p_{2}}(z)=\tilde{p_{2} \mid z=\eta}-\varepsilon \beta_{0}\left(z-\eta^{0}\right) .
$$

We use the interface condition (A.8) and (A.14) to get the value of the pressure at the interface, so finally

$$
\tilde{p_{2}}(z)=\varepsilon \frac{r}{\nu_{02}} \frac{1}{F r_{1}^{2}} h_{1}^{0}-\varepsilon \beta_{0}\left(z-\eta^{0}\right) .
$$

Thus

$$
\nabla_{x} \tilde{p_{2}}=\varepsilon \frac{r}{\nu_{02}} \frac{1}{F r_{1}^{2}} \nabla_{x} h_{1}^{0}+\varepsilon \beta_{0} \nabla_{x} \eta^{0}=\varepsilon \frac{1}{\nu_{02}} \frac{1}{F r_{1}^{2}}\left(r \nabla_{x} h_{1}^{0}+\nabla_{x} \eta^{0}\right),
$$

that does not depend on $z$.

Next in order to get an expression for the velocity $u_{2}$, we integrate twice equation (A.18). We use condition (A.12) and take into account that $\mu_{2}$ is not constant, so

$$
\mu_{2} \partial_{z} \tilde{u_{2}}=\frac{\varepsilon}{\nu_{02}} \operatorname{fric}_{f_{0}}+\nabla_{x} \tilde{p_{2}}\left(z-\eta_{f}\right)
$$

and then

$$
\tilde{u}_{2}(z)=\tilde{u_{2} \mid z=\eta}+\frac{\varepsilon}{\nu_{02}} \operatorname{fric}_{f_{0}} \int_{\eta}^{z} \frac{1}{\mu_{2}} \mathrm{~d} z+\nabla_{x} \tilde{p_{2}} \int_{\eta}^{z} \frac{z-\eta_{f}}{\mu_{2}} \mathrm{~d} z .
$$


Since we are interested in the principal order approximation $u_{2}^{0}$, we neglect the two last terms - remember that $\nabla_{x} \tilde{p_{2}} \sim \mathcal{O}(\varepsilon)$ from equation (A.24)-. So we approximate

$$
u_{2}(z)=u_{2 \mid z=\eta}+\mathcal{O}(\varepsilon)
$$

and then $u_{2}^{0}=u_{2 \mid z=\eta}$.

If we use the boundary condition at the interface (A.10) and the previous expression for $\mu_{2} \partial_{z} \tilde{u_{2}}$ evaluated in $z=\eta$ we get a relation between the friction forces:

$$
r \text { fric }_{0}=\text { fric }_{f_{0}}+\frac{h_{m}^{0}}{F r_{1}^{2}}\left(r \nabla_{x} h_{1}^{0}+\nabla_{x} \eta^{0}\right)
$$

where we have used (A.24).

At this moment, in order to find an expression for $u_{2 \mid z=\eta}$, and then for $u_{2}^{0}$, we must explicit the friction terms.

For the friction at the level $z=\eta_{f}$, we consider a Coulomb friction law (2.2). To be consistent with the development done before, the asymptotic assumption for $\delta$ the intergranular Coulomb friction angle must be

$$
\tan \delta=\varepsilon \tan \delta_{0}
$$

Developing this expression and using (A.14) we have:

$$
\operatorname{fric}_{f_{0}}=-\frac{r-1}{F r_{1}^{2}} \operatorname{sgn}\left(u_{2}^{0}\right) \tan \delta_{0} h_{m}^{0} .
$$

We consider two possible friction laws for the level $z=\eta$ and we derive the corresponding models.

\section{- Linear friction law at $z=\eta$}

We use a generalized law based on the work [41] (see Rem. A.1), that reads

$$
\text { fric }=C\left(u_{1}-u_{2}\right)_{\mid z=\eta}
$$

Remark A.1. The friction law introduced in (A.28) is based on the work performed in [41]. If we take into account the asymptotic regime considered for the viscosities, the coefficient $C$ can be written (up to second order) as:

$$
C=k h_{m}
$$

We must also take into account the adimensionalization for this friction term. Thus we assume the following dimension and asymptotic to the coefficient $C$ :

$$
C=U C^{*} ; \quad C^{*}=\varepsilon C^{0}
$$

Then we have

$$
\text { fric }_{0}=C^{0}\left(u_{1}^{0}-\varepsilon^{2} u_{2 \mid z=\eta}\right)
$$

From this expression and using (A.26) we get the value of $u_{2}^{0}$

$$
\begin{aligned}
u_{2}^{0}=u_{2 \mid z=\eta} & =\frac{1}{\varepsilon^{2}} u_{1}^{0}-\frac{1}{\varepsilon^{2} C^{0}} \text { fric }_{0} \\
& =\frac{1}{\varepsilon^{2}} u_{1}^{0}-\frac{1}{r \varepsilon^{2} C^{0}}\left(\operatorname{fric}_{f_{0}}+\frac{h_{m}^{0}}{F r_{1}^{2}}\left(r \nabla_{x} h_{1}^{0}+\nabla_{x} \eta^{0}\right)\right) \\
& =\frac{1}{\varepsilon^{2}} u_{1}^{0}-\frac{1}{r \varepsilon^{2} C^{0}} \frac{1}{F r_{1}^{2}} h_{m}^{0}\left((1-r) \operatorname{sgn}\left(u_{2}^{0}\right) \tan \delta_{0}+\left(r \nabla_{x} h_{1}^{0}+\nabla_{x} \eta^{0}\right)\right) .
\end{aligned}
$$

Now we use the relation (A.21) to write an equation for $h_{m}$ up to first order:

$$
\partial_{t_{2}} h_{m}^{0}+\operatorname{div}_{x}\left(\frac{1}{\varepsilon^{2}} h_{m}^{0} u_{1}^{0}-\frac{1}{r \varepsilon^{2} C^{0}} \frac{\left(h_{m}^{0}\right)^{2}}{F r_{1}^{2}}\left((1-r) \operatorname{sgn}\left(u_{2}^{0}\right) \tan \delta_{0}+\left(r \nabla_{x} h_{1}^{0}+\nabla_{x} \eta^{0}\right)\right)\right)=T_{m} .
$$


- Quadratic friction law at $z=\eta$

We consider now a quadratic law as follows

$$
\text { fric }=C_{1}\left|\left(u_{1}-u_{2}\right)_{\mid z=\eta}\right|\left(u_{1}-u_{2}\right)_{\mid z=\eta}
$$

with $C_{1}$ an adimensional constant that we suppose of order $\varepsilon$, that is, $C_{1}=\varepsilon C_{1}^{0}$. Then,

$$
\text { fric }_{0}=C_{1}^{0}\left|u_{1}^{0}-\varepsilon^{2} u_{2 \mid z=\eta}\right|\left(u_{1}^{0}-\varepsilon^{2} u_{2 \mid z=\eta}\right) .
$$

We can find a solution for this equation, given by:

$$
u_{1}^{0}-\varepsilon^{2} u_{2 \mid z=\eta}=\frac{1}{\sqrt{C_{1}^{0}}} \mid \text { fric }\left._{0}\right|^{1 / 2} \operatorname{sgn}\left(\text { fric }_{0}\right)
$$

So we write the value of the velocity $u_{2}^{0}$ as follows:

$$
\begin{aligned}
u_{2}^{0}=u_{2 \mid z=\eta} & =\frac{1}{\varepsilon^{2}} u_{1}^{0}-\frac{1}{\varepsilon^{2} \sqrt{C_{1}^{0}}} \mid \text { fric }\left._{0}\right|^{1 / 2} \operatorname{sgn}\left(\text { fric }_{0}\right) \\
& =\frac{1}{\varepsilon^{2}} u_{1}^{0}-\frac{1}{\varepsilon^{2}} \frac{\left(h_{m}^{0}\right)^{1 / 2}}{\sqrt{r C_{1}^{0}} F r_{1}}|\mathcal{P}|^{1 / 2} \operatorname{sgn}(\mathcal{P})
\end{aligned}
$$

with

$$
\mathcal{P}=\nabla_{x}\left(r h_{1}+h_{2}+b\right)+(1-r) \operatorname{sgn}\left(u_{2}^{0}\right) \tan \delta .
$$

\section{Appendix B: Energy BALANCE}

\section{B.1: Proof of Theorem 2.4}

In this appendix we prove the result of the energy balance associated to the proposed system that is held in Theorem 2.4. Concretely, we prove that the deduced model verifies an exact entropy dissipation energy.

First we multiply the momentum equation for the layer 1 by $r u_{1}$ and we use the mass equation to obtain:

$$
\frac{r}{2} \partial_{t}\left(g h_{1}^{2}+h_{1}\left|u_{1}\right|^{2}\right)+r \operatorname{div}_{x}\left(h_{1} u_{1}\left(\frac{\left|u_{1}\right|^{2}}{2}+g h_{1}\right)\right)+\underbrace{r g h_{1} u_{1} \nabla_{x}\left(b+h_{2}\right)}_{(a)}+\underbrace{g h_{m} u_{1} \mathcal{P}}_{(b)}=0
$$

where $\mathcal{P}=\mathcal{P}_{1}+\mathcal{P}_{2}$ with $\mathcal{P}_{1}=\nabla_{x}\left(r h_{1}+h_{2}+b\right)$ and $\mathcal{P}_{2}=(1-r) \operatorname{sgn}\left(u_{2}\right) \tan \delta$.

We use the definition of $\mathcal{P}$ to decompose the term $(b)$ in two parts, $(b)=(b)_{1}+(b)_{2}$ :

$$
\begin{gathered}
(b)_{1}=g h_{m} u_{1} \mathcal{P}_{1}=g h_{m} u_{1} \nabla_{x}\left(r h_{1}+h_{2}+b\right) \\
(b)_{2}=g h_{m} u_{1} \mathcal{P}_{2}=g h_{m} u_{1}(1-r) \operatorname{sgn}\left(u_{2}\right) \tan \delta .
\end{gathered}
$$

Now we multiply the mass conservation equation for the layer 2 by $\tilde{\mathcal{P}}_{1}=g\left(r h_{1}+h_{2}+b\right)$. Note that $\nabla_{x} \tilde{\mathcal{P}}_{1}=g \mathcal{P}_{1}$.

- For the linear friction law case,

$$
\underbrace{\tilde{\mathcal{P}}_{1} \partial_{t} h_{2}}_{(c)}+\underbrace{\tilde{\mathcal{P}}_{1} \operatorname{div}_{x}\left(h_{m} u_{1}\right)}_{(d)}-\underbrace{\tilde{\mathcal{P}}_{1} \operatorname{div}_{x}\left(h_{m} \frac{\vartheta}{1-r} \mathcal{P} \sqrt{(1 / r-1) g d_{s}}\right)}_{\left(e^{L}\right)}=0 ;
$$


- for the quadratic friction law case

$$
\underbrace{\tilde{\mathcal{P}}_{1} \partial_{t} h_{2}}_{(c)}+\underbrace{\tilde{\mathcal{P}}_{1} \operatorname{div}_{x}\left(h_{m} u_{1}\right)}_{(d)}-\underbrace{\tilde{\mathcal{P}}_{1} \operatorname{div}_{x}\left(h h_{m} \sqrt{\frac{\vartheta}{1-r}}|\mathcal{P}|^{1 / 2} \operatorname{sgn}(\mathcal{P}) \sqrt{(1 / r-1) g d_{s}}\right)}_{\left(e^{Q}\right)}=0 .
$$

Now, after some simple calculations, the terms $(a)$ and $(c)$ gives:

$$
(a)+(c)=\frac{1}{2} g \partial_{t}\left(\left(b+h_{2}\right)^{2}\right)+\operatorname{div}_{x}\left(r g h_{1}\left(b+h_{2}\right) u_{1}\right)+r g \partial_{t}\left(h_{1}\left(b+h_{2}\right)\right)
$$

and we also directly obtain

$$
(b)_{1}+(d)=\operatorname{div}_{x}\left(h_{m} u_{1} \tilde{\mathcal{P}}_{1}\right) .
$$

Finally, the last terms read

$$
\left(e^{L}\right)=-\operatorname{div}_{x}\left(h_{m} \frac{\vartheta}{1-r} \tilde{\mathcal{P}}_{1} \mathcal{P} \sqrt{(1 / r-1) g d_{s}}\right)+g h_{m} \frac{\vartheta}{1-r} \sqrt{(1 / r-1) g d_{s}} \mathcal{P} \mathcal{P}_{1} .
$$

We sum the last term in this expression of $\left(e^{L}\right)$ with $(b)_{2}$ :

$$
\begin{aligned}
& g h_{m} u_{1}(1-r) \operatorname{sgn}\left(u_{2}\right) \tan \delta+g h_{m} \frac{\vartheta}{1-r} \sqrt{(1 / r-1) g d_{s}} \mathcal{P}\left(\mathcal{P}-\mathcal{P}_{2}\right) \\
& =g h_{m} \frac{\vartheta}{1-r} \sqrt{(1 / r-1) g d_{s}}|\mathcal{P}|^{2}+g h_{m}(1-r) \tan \delta \operatorname{sgn}\left(u_{2}\right)\left(u_{1}-\frac{\vartheta}{1-r} \sqrt{(1 / r-1) g d_{s}} \mathcal{P}\right) \\
& =g h_{m} \frac{\vartheta}{1-r} \sqrt{(1 / r-1) g d_{s}}|\mathcal{P}|^{2}+g h_{m}(1-r) \tan \delta \sqrt{(1 / r-1) g d_{s}}\left|u_{2}\right|
\end{aligned}
$$

where for the last identity we have taken into account the definition of the velocity given in (2.10).

We follow the same process for $\left(e^{Q}\right)$ :

$$
\begin{aligned}
\left(e^{Q}\right)= & -\operatorname{div}_{x}\left(h_{m} \sqrt{\frac{\vartheta}{1-r}} \tilde{\mathcal{P}}_{1}|\mathcal{P}|^{1 / 2} \operatorname{sgn}(\mathcal{P}) \sqrt{(1 / r-1) g d_{s}}\right) \\
& +g h_{m} \sqrt{\frac{\vartheta}{1-r}} \sqrt{(1 / r-1) g d_{s}}|\mathcal{P}|^{1 / 2} \operatorname{sgn}(\mathcal{P}) \mathcal{P}_{1}
\end{aligned}
$$

And now, in the same manner than before and using (2.11), this last term together with $(b)_{2}$ gives:

$$
g h_{m} \sqrt{\frac{\vartheta}{1-r}} \sqrt{(1 / r-1) g d_{s}}|\mathcal{P}|^{3 / 2}+g h_{m}(1-r) \tan \delta \sqrt{(1 / r-1) g d_{s}}\left|u_{2}\right| .
$$

So, finally we obtain (2.34) and (2.35), where the right hand side terms in both cases are non-positive.

\section{B.2: Proof of Theorem 2.6}

In this section we analyze the energy associated to a general Saint-Venant-Exner model for a modified friction in the momentum equation of the Saint-Venant system:

$$
\hat{\tau} / \rho_{1}=\tau / \rho_{1}+\frac{1}{r} \xi_{m} \sqrt{g \zeta\left(h_{1}\right)} \mathcal{R} \quad \text { with } \quad \mathcal{R}=g \nabla_{x}\left(r h_{1}+h_{2}+b\right) .
$$

This model is given in equations (1.1)-(1.4) that we remind next:

$$
\left\{\begin{array}{l}
\partial_{t} h_{1}+\operatorname{div}_{x} q_{1}=0 \\
\partial_{t} q_{1}+\operatorname{div}_{x}\left(\frac{\left|q_{1}\right|^{2}}{h_{1}}+\frac{1}{2} g h_{1}^{2}\right)+g h_{1} \nabla_{x}\left(h_{2}+b\right)+\hat{\tau} / \rho_{1}=0 \\
\partial_{t} h_{2}+\operatorname{div}_{x} q_{b}=0
\end{array}\right.
$$


where

$$
\begin{gathered}
\frac{q_{b}}{Q}=\frac{1}{1-\varphi} \operatorname{sgn}(\tau) k_{1} \theta^{m_{1}}\left(\theta-k_{2} \theta_{c}\right)_{+}^{m_{2}}\left(\sqrt{\theta}-k_{3} \sqrt{\theta_{c}}\right)_{+}^{m_{3}}, \\
Q=d_{s} \sqrt{g(1 / r-1) d_{s}}, \quad \theta=\frac{|\tau| d_{s}^{2}}{g\left(\rho_{2}-\rho_{1}\right) d_{s}^{3}} \quad \text { and } \tau=\rho_{1} g \zeta\left(h_{1}\right) u_{1}\left|u_{1}\right| .
\end{gathered}
$$

To prove Theorem 2.6 we follow the same development above. First, we write the discharge $q_{b}$ in the following way:

$$
\frac{q_{b}}{Q}=\frac{\xi_{m}}{d_{s}} \operatorname{sgn}(\tau) \sqrt{\theta}
$$

where

$$
\frac{\xi_{m}}{d_{s}}=\frac{1}{1-\varphi} k_{1} \theta^{m_{1}-1 / 2}\left(\theta-k_{2} \theta_{c}\right)_{+}^{m_{2}}\left(\sqrt{\theta}-k_{3} \sqrt{\theta_{c}}\right)_{+}^{m_{3}} .
$$

Now, from the definition of $\theta$ and $\tau$ we write:

$$
\sqrt{\theta}=\frac{\sqrt{g \zeta\left(h_{1}\right)}\left|u_{1}\right|}{\sqrt{g(1 / r-1) d_{s}}}=\operatorname{sgn}(\tau) \frac{\sqrt{g \zeta\left(h_{1}\right)}}{\sqrt{g(1 / r-1) d_{s}}} u_{1} .
$$

So, by using the definition of $\xi_{m}$ we can rewrite $q_{b}$ as follows:

$$
q_{b}=\xi_{m} \sqrt{g \zeta\left(h_{1}\right)} u_{1} .
$$

Note that we have rewritten the evolution equation for the sediment in a different manner but no modification has been introduced in it. As we mentioned above, the only modification needed to obtain the dissipative energy balance is taken into account in the friction term in the momentum conservation equation of the Saint-Venant system.

First, we multiply the momentum equation of the first layer by $r u_{1}$ and we use the mass equation to obtain:

$$
\begin{aligned}
\frac{r}{2} \partial_{t}\left(g h_{1}^{2}+h_{1}\left|u_{1}\right|^{2}\right) & +r \operatorname{div}_{x}\left(h_{1} u_{1}\left(\frac{\left|u_{1}\right|^{2}}{2}+g h_{1}\right)\right)+\underbrace{r g h_{1} u_{1} \nabla_{x}\left(b+h_{2}\right)}_{(a)} \\
& +r g \zeta\left(h_{1}\right)\left|u_{1}\right|^{3}+\underbrace{\xi_{m} \sqrt{g \zeta\left(h_{1}\right)} \mathcal{R} u_{1}}_{(b)}=0
\end{aligned}
$$

Now we multiply the equation for layer 2 by $\tilde{\mathcal{R}}=g\left(r h_{1}+h_{2}+b\right)$, where $\nabla_{x} \tilde{\mathcal{R}}=\mathcal{R}$. Then,

$$
\underbrace{\tilde{\mathcal{R}} \partial_{t} h_{2}}_{(c)}+\underbrace{\tilde{\mathcal{R}} \operatorname{div}_{x}\left(\xi_{m} \sqrt{g \zeta\left(h_{1}\right)} u_{1}\right)}_{(d)}=0 .
$$

The terms $(a)$ and $(c)$ combine in the same manner than before and

$$
(b)+(d)=\operatorname{div}_{x}\left(\xi_{m} u_{1} \sqrt{g \zeta\left(h_{1}\right)} \tilde{\mathcal{R}}\right) .
$$

Then, (2.39) is satisfied.

Acknowledgements. This research has been partially supported by the Spanish Government and FEDER through the Research project MTM2012-38383-C02-02, and by the Andalusian Government through the projects P11- RNM7069 and P11-FQM8179. Authors would like to thank to Pierre-Yves Lagrée for his comments and the interesting discussions that followed during his visit to the University of Seville in November 2014. 


\section{REFERENCES}

[1] K. Ashida and M. Michiue, Study on hydraulic resistance and bedload transport rate in alluvial streams. JSCE Tokyo 206 (1972) 59-69.

[2] P. Aussillous, J. Chauchat, M. Pailha, M. Médale and E. Guazzelli, Investigation of the mobile granular layer in bed-load transport. J. Fluid Mech. 736 (2013) 594-615.

[3] R.A. Bagnold, The Flow of Cohesionless Grains in Fluids. Roy. Soc. London Philos. Trans. Ser. A: Math. Phys. Sci. 249 (1956) 235-297.

[4] R.A. Bagnold, An approach to the sediment transport problem from general physics. In: Physical of Sediments and Transport by Wind and Water. Amer. Society of Civil Engineers (1988).

[5] C. Cassar, M. Nicolas and O. Pouliquen, Submarine granular flows down inclined planes. Phys. Fluids 17 (2005) 103301.

[6] M.J. Castro Díaz, E.D. Fernández-Nieto and A.M. Ferreiro, Sediment transport models in Shallow Water equations and numerical approach by high order finite volume methods. Comput. Fluids 37 (2008) 299-316.

[7] J. Chauchat and M. Médale, A 3D numerical model for incompress-ible two-phase flow of a granular bed submitted to a laminar shearing flow. Comput. Meth. Appl. Mech. Eng. 199 (2010) 439-449.

[8] F. Charru, Interface boundary value problem for the Navier-Stokes equations in thin domains. J. Differ. Eq. 208 (2005) 449-493.

[9] F. Charru, Selection of the ripple length on a granular bed sheared by a liquid flow. Phys. Fluids 18 (2006) 121508.

[10] I.D. Chueskov, G. Raugel and A.M. Rekalo, Selection of ripple length on a granular bed sheared by a liquid flow. Phys. Fluids 18 (2006) 121508

[11] M. Church, P. Biron and A. Roy, Gravel Bed Rivers: Processes, Tools, Environments. John Wiley \& Sons (2012).

[12] G. Cimatti, A Rigorous Justification of the Reynolds Equation. Quart. Appl. Math. XLV 4 (1987) 627-644.

[13] M. Colombini, Revisiting the linear theory of sand dune formation. J. Fluid Mech. 502 1-6 (2004).

[14] W.F. Cope, The hydrodynamic theory of film lubrication. Proc. R. Soc. London, Ser. A 197 (1949) $201-217$.

[15] S. Cordier, C. Lucas and J.D.D. Zabsonré, A two time-scale model for tidal bed-load transport. Commun. Math. Sci. 10 (2012) 875-888.

[16] H.A. Einstein, Formulas for the transportation of bedload. ASCE 107 (1942) 561-575.

[17] F. Exner, Uber die wechselwirkung zwischen wasser und geschiebe in flussen, Sitzungsber. Akad. Wissenschaften pt. IIa. Bd. 134 (1925).

[18] F. Engelund and J. Dresoe. A sediment transport model for straight alluvial channels. Nordic Hydrol. 7 (1976) $293-306$.

[19] F. Engelund and E. Hansen, A monograph on sediment transport in alluvial streams. Danish Technical Press, Copenhagen (1967).

[20] F. Luque and V. Beek, Erosion and transport of bedload sediment. J. Hydraulaul. Res. 14 (1976) $127-144$.

[21] E.D. Fernández-Nieto, Modelling and numerical simulation of submarine sediment shallow flows: transport and avalanches. SeMA J. 49 (2009) 83-103.

[22] E.D. Fernández-Nieto, F. Bouchut, D. Bresch, J.M. Castro and A. Mangeney, A new-Savage-Hutter type model for submarine avalanches and genrated tsunami. J. Comput. Phys. 227 (2008) 7720-7754.

[23] E.D. Fernández-Nieto, G. Narbona-Reina and J.D.D. Zabsonré, Formal derivation of a bilayer model coupling Shallow Water and Reynolds equations: evolution of a thin pollutant layer over the water. Eur. J. Appl. Math. 24 (2013) $803-833$.

[24] E.D. Fernández-Nieto, C. Lucas, T. Morales de Luna and S. Cordier, On the influence of the thickness of the sediment moving layer in the definition of the bedload trasport formula in Exner systems. Comp. Fluids 91 (2014) 87-106.

[25] Y. Forterre and O. Pouliquen, Flows of dense granular media. Ann. Rev. Fluid Mech. 40 (2008) 1-24.

[26] A.C. Fowler, N. Kopteva and C. Oakley, The formation of river channel. SIAM J. Appl. Math. 67 (2007) 1016-1040.

[27] J. Fredsøe, On the development of dunes in erodible channel. J. Fluid Mech. 64 (1974) 1-16.

[28] G. Garegnani, G. Rosatti and L. Bonaventura, Free surface flows over mobile bed: mathematical analysis and numerical modeling of coupled and decoupled approaches. Commun. Appl. Ind. Math. 2 (2011).

[29] A.J. Grass, Sediments transport by waves and currents. SERC London Cent. Mar. Technol., Report No. FL29 (1981).

[30] F. Gerbeau and B. Perthame, Derivation of viscous Saint-Venant system for laminar Shallow-Water; Numerical validation. Disc. Cont. Dynam. Syst. Series B 1 (2001) 89-102.

[31] R. Jackson, The Dynamics of Fluidized Particles. Cambridge University Press, Cambridge, (2000).

[32] A.A. Kalinske, Criteria for determining sand transport by surface creep and saltation. Trans. AGU. 23 (1942) 639-643.

[33] A. Kovacs and G. Parker. A new vectorial bedload formulation and its application to the time evolution of straight river channels. J. Fluid Mech. 267 (1994) 153-183.

[34] K.K.J. Kouakou and P.Y. Lagrée, Evolution of a model dune in a shear flow. Eur. J. Mech. B/Fluids 25 (2006) $348-359$.

[35] P.Y. Lagrée, L. Staron and S. Popinet The granular column collapse as a continuum: validity of a two-dimensional Navier-Stokes model with a $\mu(I)$-rheology. J. Fluid. Mech. 686 (2011) 378-408.

[36] D.K. Lysne, Movement of sand in tunnels. Proc. A.S.C.E. 95 (1969) 1835-1846.

[37] F. Marche, Theoretical and numerical study of Shallow Water models. Applications to Nearshore hydrodynamics. Ph.D. thesis, University of Bordeaux (2005).

[38] Meyer-Peter and R. Müller, Formulas for bed-load transport. Rep. 2nd Meet. Int. Assoc. Hydraul. Struct. Res., Stockolm 1948. ASCE. 107 (1942) 561-575. 
[39] T. Morales De Luna, J.M. Castro-Díaz and C. Parés-Madroñal, A Duality Method for Sediment Transport Based on a Modified Meyer-Peter and Müller Model. J. Sci. Comput. 48 (2009) 258-273.

[40] T. Morales De Luna, J.M. Castro-Díaz, C. Parés-Madroñal and E.D. Fernández-Nieto, On a Shallow Water Model for the Simulation of Turbidity Currents. Commun. Comput. Phys. 6 (2011) 4848-882.

[41] G. Narbona-Reina, J.D. Zabsonré, E.D. Fernández-Nieto and D. Bresch, Derivation of a bi-layer Shallow-Water model with viscosity. Numerical validation. CMES 43 (2009) 27-71.

[42] P. Nielsen, Coastal Bottom Boundary Layers and Sediment Transport. World Scientific Publishing, Singapore. Adv. Ser. Ocean Eng. 4 (1992).

[43] A. Oron, S.H. Davis and S.G. Bankoff, Long scale evolution of thin films. Rev. Mod. Phys. 69 (1997).

[44] M. Ouriemi, P. Aussillous and E. Guazzelli, Sediment dynamics. Part I: Bed-load transport by shearing flows. J. Fluid Mech. 636 (2009) 295-319.

[45] M. Pailha, J. Chauchat, P. Aussillous, M. Médale and E. Guazzelli. Bed-load Transport. Part 2: The Mobile Granular Layer. Ph.D. thesis (2011).

[46] G. Parker, G. Seminara and L. Solari, Bedload at low Shields stress on arbitrarily sloping beds: Alternative entrainment formulation. Water Resources Res. 29 (2003) 7.

[47] C. Parés and M.J. Castro, On the well-balance property of Roe's method for nonconservative hyperbolic systems. Applications to shallow-water systems. ESAIM: M2AN 38 (2004) 821-852.

[48] E. Peña González, Estudio numérico y experimental del transporte de sedimentos en cauces aluviales. Ph.D. thesis, Universidade da Coruña, Grupo de Ingeniería del agua y del medio ambiente (2002).

[49] T. Revil-Baudard and J. Chauchat, A two-phase model for sheet flow regime based on dense granular flow rheology. J. Geophys. Res. Oceans 118 (2013) 619-634.

[50] M. Peybernes, Analyse de problème mathématiques de la mécanique des fluides de type bi-couche et à frontière libre. Ph.D. thesis, University of Pascal Paoli (2006).

[51] O. Reynolds, On the theory of lubrication and its application to Mr. Beauchamp Tower's experiment. Phil. Trans. R. Soc. London Part I (1886) 228-310.

[52] L.C. Van Rijn, Sediment transport (I): bedload transport. J. Hydraul. Div. Proc. ASCE 110 (1984) $1431-56$.

[53] P.C. Roos, Seabed Pattern Dynamics and Offshore Sand Extraction. Ph.D. thesis, University of Twente (2004).

[54] H. Schuttelaars, Evolution and Stability Analysis of Bottom Patterns in Tidal Embayements. Ph.D. thesis, Utrecht University (1997).

[55] G. Seminara, L. Solari and G. Parker, Bedload at low Shields stress on arbitrarily sloping beds: Failure of the Bagnold hypothesis. Water Resources Res. 38 (2002) 11.

[56] N. Struiksma. K.W. Olesen. C. Flokstra and H.J. De Vriend, Bed deformation in curved alluvial channels. J. Hudraulic Res. 23 (1985) 57-79.

[57] P. Tassi, S. Rhebergen, C. Vionnet and O. Bokhove, A discontinuous Galerkin finite element model for morphodynamical evolution in shallow flows. Comp. Meth. App. Mech. Eng. 197 (2008) 2930-2947.

[58] H.J. de Vriend, J. Zyserman, J. Nicholson, J.A. Roelvink, P. Péchon and H.N. Southgate, Medium-term 2DH coastal area modelling. Coastal Engineering 21 (1993) 193-224.

[59] J.D. Zabsonré, Modèles visqueux en sédimentation et stratification: obtention formelle, stabilité théorique et schémas volumes finis bien équilibrés. Ph.D. thesis, Université de Savoie (2008).

[60] J.D.D. Zabsonré, C. Lucas and E.D. Fernández-Nieto, An energetically consistent viscous sedimentation model. Math. Models Methods Appl. Sci. 19 (2009) 477-499. 